\title{
THE ANNIHILATOR OF THE LEFSCHETZ MOTIVE
}

\author{
INNA ZAKHAREVICH
}

\begin{abstract}
In this paper we study a spectrum $K\left(\mathcal{V}_{k}\right)$ such that $\pi_{0} K\left(\mathcal{V}_{k}\right)$ is the Grothendieck ring of varieties and such that the higher homotopy groups contain more geometric information about the geometry of varieties. We use the topology of this spectrum to analyze the structure of $K_{0}\left[\mathcal{V}_{k}\right]$ and show that classes in the kernel of multiplication by $\left[\mathbb{A}^{1}\right]$ can always be represented as $[X]-[Y]$, where $[X] \neq[Y], X \times \mathbb{A}^{1}$ and $Y \times \mathbb{A}^{1}$ are not piecewise isomorphic, but $\left[X \times \mathbb{A}^{1}\right]=\left[Y \times \mathbb{A}^{1}\right]$ in $K_{0}\left[\mathcal{V}_{k}\right]$. Along the way we present a new proof of the result of Larsen-Lunts on the structure on $K_{0}\left[\mathcal{V}_{k}\right] /\left(\left[\mathbb{A}^{1}\right]\right)$.
\end{abstract}

The Grothendieck ring of varieties over a field $k$-denoted $K_{0}\left[\mathcal{V}_{k}\right]$-is defined to be the free abelian group generated by varieties over $k$, modulo the relation

$$
[X]=[Y]+[X \backslash Y] \quad \text { for } Y \text { a closed subvariety of } X \text {. }
$$

The ring structure is given by the formula $[X][Y]=[X \times Y]$. This ring was first introduced by Grothendieck in a letter to Serre in 1964, and has since appeared in various places in the study of motivic integration and birational geometry. (For more on the Grothendieck ring, see for example [LL03, [LS12, [LS10.) This ring is quite complicated; for example, it is not an integral domain ( $\mathrm{Poo02}]$ ).

There are two important structural questions about $K_{0}\left[\mathcal{V}_{k}\right]$. The Grothendieck ring of varieties comes with a filtration on the generators given by the dimension of the variety, where the $n$ th graded piece of the filtration is given by those elements that can be represented as a formal sum of varieties of dimension at most $n$. Equivalently, the $n$-th graded piece is the image of the homomorphism

$$
\psi_{n}: \mathbb{Z}[X \mid \operatorname{dim} X \leq n] /([X]=[Y]+[X \backslash Y]) \longrightarrow K_{0}\left[\mathcal{V}_{k}\right]
$$

In [LL03, Question 1.2] (and, equivalently, in [Gro99, p121]) the following question is asked:

Question 1. Is $\psi_{n}$ injective? Equivalently, do all birational automorphisms of varieties extend to piecewise isomorphisms?

By a piecewise isomorphism between varieties $X$ and $Y$ we mean a pair of stratifications of $X$ and $Y$ and isomorphisms of corresponding strata. By the definition of $K_{0}\left[\mathcal{V}_{k}\right]$, if $X$ and $Y$ are piecewise isomorphic then $[X]=[Y]$ in $K_{0}\left[\mathcal{V}_{k}\right]$; the question is interested in the converse of this statement. In recent work of Borisov [Bor] and Karzhemanov [Kar] counterexamples to the injectivity of $\psi_{n}$ are constructed when $k$ is a subfield of $\mathbf{C}$, and we expect that in fact it will not be injective over any $k$. The associated graded of the filtration induced on $K_{0}\left[\mathcal{V}_{k}\right]$ by the images of $\psi_{n}$ is still unknown.

The second structural question concerns multiplication by the Lefschetz motive $\mathbb{L} \stackrel{\text { def }}{=}\left[\mathbb{A}^{1}\right]$. In Kontsevich's definition of the motivic integral, the motivic measure has values in $K_{0}\left[\mathcal{V}_{k}\right]$, but is only well-defined up to a power of $\mathbb{L}$. Thus in order for motivic integration to be well-defined it is necessary to invert $\mathbb{L}$ and consider the ring $K_{0}\left[\mathcal{V}_{k}\right]\left[\mathbb{L}^{-1}\right]$. (For more on motivic integration, see for example [DL99].) This leads to the following question.

\section{Question 2. Is $\mathbb{L}$ a zero divisor?}

This question was also recently resolved by Borisov in [Bor]. In fact, Borisov's main result was to construct an element in the kernel of multiplication by $\mathbb{L}$, and, seemingly coincidentally, his method also constructed an element in the kernel of $\psi_{n}$. A precise algebraic description of the kernel of 
multiplication by $\mathbb{L}$, and, more interestingly, of the kernel of the localization $K_{0}\left[\mathcal{V}_{k}\right] \longrightarrow K_{0}\left[\mathcal{V}_{k}\right]\left[\mathbb{L}^{-1}\right]$, is still unknown.

In this paper we propose to replace the Grothendieck ring of varieties with a spectrum $K\left(\mathcal{V}_{k}\right)$, which we call the Grothendieck spectrum of varieties 1 . This spectrum has the property that $\pi_{0} K\left(\mathcal{V}_{k}\right) \cong K_{0}\left[\mathcal{V}_{k}\right]$, and that its higher homotopy groups contain further geometric information about the decomposition of varieties. This spectrum encodes additional geometric information which is lost when passing to the Grothendieck ring. We use topological tools to study the structure of this spectrum and illuminate a precise relationship between Questions 1 and 2 ,

The spectrum $K\left(\mathcal{V}_{k}\right)$ also comes with a dimension filtration which comes from the dimension of the varieties which "generate" the spectrum. Although the associated graded of $K_{0}\left[\mathcal{V}_{k}\right]$ is unknown, it turns out that the associated graded of this spectrum has a beautifully simple description.

Theorem A. The category $\mathcal{V}_{k}$ of varieties over $k$ and locally closed inclusions comes with a filtration, where the $n$-th filtered piece $\mathcal{V}_{k}^{(n)}$ is generated by varieties of dimension $n$. This filtration gives rise to a filtration on $K\left(\mathcal{V}_{k}\right)$, whose $n$-th graded piece is

$$
\bigvee_{[X] \in B_{n}}\left(\Sigma^{\infty} \mathrm{BAut}_{k} k(X)_{+}\right)
$$

where $B_{n}$ is the set of birational isomorphism classes of varieties of dimension $n$ and Aut $_{k} k(X)$ is the group of birational automorphisms of the variety $X$.

This theorem implies that there exists a spectral sequence in stable homotopy

$$
E_{p, q}^{1}=\bigoplus_{[X] \in B_{q}} \pi_{p}^{s} B \operatorname{Aut}_{k} k(X) \oplus \pi_{p}^{s} S^{0} \Longrightarrow \pi_{p} K\left(\mathcal{V}_{k}\right)
$$

in particular, the $p=0$ column converges to $K_{0}\left[\mathcal{V}_{k}\right] 2$ By analyzing the construction of $K\left(\mathcal{V}_{k}\right)$ we can compute the differentials between the 1-st and 0 -th columns in the spectral sequence to get the following:

Theorem B. There exist nonzero differentials between the 1-st and 0 -th column of some page of the spectral sequence if and only if $\psi_{n}$ has a nonzero kernel for some $n$.

This theorem holds over all fields $k$. To prove this we show that the spectral sequence constructed above produces an obstruction theory for birational automorphisms extending to piecewise isomorphisms, therefore answering the question of Gromov in Gro99, p121]; this is proved in more detail in Theorem 3.4. At first it may seem that this does not give very much information about $\psi_{n}$, since the birational automorphism groups of varieties are very complex. However, this repackaging allows us to see a clear connection between the answers to Questions 1 and 2. In order to make these conclusions, though, we need to restrict to "convenient" fields $k$. At the moment, only fields with characteristic 0 are known to be convenient. For more details, see Definition 5.1

Theorem C. Suppose that $k$ is a convenient field. If $\mathbb{L}$ is a zero divisor in $K_{0}\left[\mathcal{V}_{k}\right]$ then $\psi_{n}$ is not injective for some $n$.

Moreover, we can say something about the kernel of multiplication by $\mathbb{L}$ :

Theorem D. Suppose that $k$ is a convenient field. If $\chi$ is in the kernel of multiplication by $\mathbb{L}$ then we can represent $\chi$ as $[X]-[Y]$ where $\left[X \times \mathbb{A}^{1}\right]=\left[Y \times \mathbb{A}^{1}\right]$ but $X \times \mathbb{A}^{1}$ and $Y \times \mathbb{A}^{1}$ are not piecewise isomorphic.

\footnotetext{
${ }^{1}$ For those readers unfamiliar with stable homotopy, almost all results in the paper still hold true if "spectrum" is replaced everywhere with "homology theory." In particular, all homotopy groups of a spectrum are abelian groups.

${ }^{2}$ This grading is inspired by the grading for the Adams Spectral Sequence, and is optimized so that our spectral sequence is a first-quadrant spectral sequence and fits comfortably on the page. See Figure 1 on page 8.
} 
These theorems tell us that Borisov's beautiful coincidence was not a coincidence at all: any element in the kernel of multiplication by $\mathbb{L}$ produces an element in the kernel of $\psi_{n}$.

The proofs of Theorems $\mathrm{C}$ and $\mathrm{D}$ are based on the following observation. We have a map of spectra $\mathbb{L}: K\left(\mathcal{V}_{k}\right) \longrightarrow K\left(\mathcal{V}_{k}\right)$ induced by multiplication by $\mathbb{L}$. If we can construct the cofiber $C$ of this map then we get an exact sequence

$$
K_{1}\left(\mathcal{V}_{k}\right) \stackrel{p r}{\longrightarrow} \pi_{1} C \longrightarrow \pi_{0} K\left(\mathcal{V}_{k}\right) \stackrel{\cdot \mathbb{L}}{\longrightarrow} \pi_{0} K\left(\mathcal{V}_{k}\right) \longrightarrow \pi_{0} C .
$$

The map $\cdot \mathbb{L}$ is exactly multiplication by $\mathbb{L}$ on $K_{0}\left[\mathcal{V}_{k}\right]$. Since this sequence is exact, the kernel of this map is the same as the cokernel of the map pr, and it turns out that this cokernel is much simpler to study than the kernel of $\mathbb{L}$. This approach also produces an alternate proof of the following result, which was originally proved in [LL03, Theorem 2.3]:

Theorem E. There is an isomorphism of abelian groups $K_{0}\left[\mathcal{V}_{\mathbf{C}}\right] /(\mathbb{L}) \longrightarrow \mathbb{Z}[S B]$, where $S B$ is the set of stable birational isomorphism classes of varieties.

Remark 0.1. The theorem of Larsen and Lunts actually claims that this is an isomorphism of rings. The techniques explained in this paper should prove that statement as well, since the spectral sequence comes with a multiplicative structure. The details of the multiplicative structure are deferred until future work.

As an amusing corollary of our techniques, we also give an extension of Liu and Sebag's result [LS10, Corollary 5(1)] that if $X$ and $Y$ are smooth projective varieties and $[X]=[Y]$ in $K_{0}\left[\mathcal{V}_{k}\right]$ then $X$ and $Y$ are stably birational; this is proved in Corollary 5.6. In our extension we show that if two varieties are the smallest-dimensional representatives of their stable birational isomorphism classes then if they are equal in the Grothendieck ring then they are stably birational, regardless of whether they are smooth or projective. Note that this is not simply a corollary of Theorem E as the homomorphism in Theorem $\mathrm{E}$ only takes smooth projective varieties to their stable birational isomorphism classes; the image of the homomorphism on a general variety is not given simply by its stable birational isomorphism class.

This paper is organized as follows. In Section 1 we introduce the technical machinery of assemblers and their $K$-theory, which are the tools necessary to define $K\left(\mathcal{V}_{k}\right)$ and prove Theorem A, In Section 2 we give a brief review of the facts about spectral sequences that we need. Section 3 concludes the analysis of the filtration on $K\left(\mathcal{V}_{k}\right)$ and proves Theorem B. Section 4 analyzes the cofiber of multiplication by $\mathbb{L}$ in general fields. Finally, Section 5 restricts to convenient fields and proves Theorems $\mathrm{C}$, D and E

Acknowledgements. The author would like to thank Dan Grayson, Mike Hopkins, Peter May, Matthew Morrow, Madhav Nori, Aaron Silberstein and Chuck Weibel for helpful discussions of this material, and Mike Hill for a very illuminating discussion during the JMM. The author would also like to thank the Institute for Advanced Study and the University of Chicago, as well as the NSF MSRFP grant for financial support.

Notation. We write $\mathbf{S p}$ for the category of spectra. In this paper, we take as our model for this category the category of symmetric spectra of simplicial sets. When a model structure is required, we take the stable structure with the levelwise cofibrations; for more detail, see Remark 1.7. All homotopy groups are stable homotopy groups; in forthcoming sections we drop the $s$ superscript. For a topological space $X$ we write $\Sigma_{+}^{\infty} X$ for the spectrum $\Sigma^{\infty}\left(X_{+}\right)$.

For any set $S$, we write $\mathbb{Z}^{\oplus S} \stackrel{\text { def }}{=} \bigoplus_{s \in S} \mathbb{Z}$. We also use the highly nonstandard notation

$$
\widetilde{\mathbb{Z}}^{\oplus S} \stackrel{\text { def }}{=} \begin{cases}\operatorname{ker}\left(\mathbb{Z}^{\oplus S} \stackrel{+}{\longrightarrow} \mathbb{Z}\right) & \text { if } S \neq \emptyset, \\ \mathbb{Z} / 2 & \text { if } S=\emptyset .\end{cases}
$$

We write $\uplus$ to refer to a union which is disjoint as sets. 


\section{INTRODUCING ASSEMBLERS}

Assemblers are introduced in [ZakA]. In this paper we quote the definition of an assembler and as few theorems as is necessary to facilitate understanding of the main results; for a more detailed exposition, see [ZakA].

Definition 1.1. An assembler is a small Grothendieck site which satisfies the following extra axioms.

(I) $\mathcal{C}$ has an initial object $\varnothing$ and the empty family is a covering family of $\varnothing$.

(R) For any object $A$ in $\mathcal{C}$, any two finite disjoint covering families of $A$ have a common refinement which is itself a finite disjoint covering family.

(M) All morphisms in $\mathcal{C}$ are monomorphisms.

We say that a family of morphisms $\left\{f_{i}: A_{i} \longrightarrow A\right\}_{i \in I}$ in an assembler $\mathcal{C}$ is disjoint if for all $i \neq j \in I$, the pullback of

$$
A_{i} \stackrel{f_{i}}{\longrightarrow} A \stackrel{f_{j}}{\longleftarrow} A_{j}
$$

exists and is equal to $\varnothing$. When $\mathcal{C}$ has all pullbacks, (R) holds automatically.

The category of assemblers, denoted Asm, has

objects: assemblers $\mathcal{C}$, and

morphisms: functors $\mathcal{C} \longrightarrow \mathcal{D}$ which are continuous with respect to the Grothendieck topology, preserve the initial object, and which take disjoint families to disjoint families.

The fundamental result about the category of assemblers is the following.

Theorem 1.2. There is a functor $K: \mathbf{A s m} \longrightarrow \mathbf{S p}$ with the following properties:

(1) The group $\pi_{0} K(\mathcal{C})$ is the free abelian group on objects of $\mathcal{C}$, under the relations that for every finite disjoint covering family $\left\{f_{i}: A_{i} \longrightarrow A\right\}_{i \in I}$ in $\mathcal{C}$,

$$
[A]=\sum_{i \in I}\left[A_{i}\right] .
$$

(2) Every element of $\pi_{1} K(\mathcal{C})$ can be represented by the following data:

- a pair of finite tuples $\left\{A_{i}\right\}_{i \in I},\left\{B_{j}\right\}_{j \in J}$ of objects in $\mathcal{C}$, and

- for $\epsilon= \pm 1$, a map of finite sets $f_{\epsilon}: I \longrightarrow J$ and for all $i \in I$, morphisms $f_{\epsilon i}: A_{i} \longrightarrow B_{f_{\epsilon}(i)}$ such that for each $j \in J$ the family $\left\{f_{\epsilon i}: A_{i} \longrightarrow B_{j}\right\}_{i \in f_{\epsilon}^{-1}(j)}$ is a covering family.

For conciseness we write $K_{i}(\mathcal{C})$ for $\pi_{i} K(\mathcal{C})$.

We purposefully leave the relations between generators in part (2) of the theorem imprecise, as this is the most general statement we need in this paper. For a more precise statement see [ZakB, Corollary 3.10].

The main example of an assembler that we are interested in in this paper is the assembler of varieties.

Example 1.3. Let $k$ be a field. The assembler $\mathcal{V}_{k}$ has as objects varieties over $k$; here by "variety" we mean a reduced separated scheme of finite type over $k$. The morphisms in $\mathcal{V}_{k}$ are locally closed embeddings. The Grothendieck topology is generated by the coverage $\{Y \hookrightarrow X, X \backslash Y \hookrightarrow X\}$ for $Y$ a closed subvariety of $X$. (For background on coverages, see for example [Joh02.)

When $k$ is clear from context we write $\mathcal{V}$ instead of $\mathcal{V}_{k}$.

Example 1.4. Let FinSet be the category of finite sets and injective morphisms. Then this is an assembler if we define a family of morphisms $\left\{f_{i}: A_{i} \longrightarrow A\right\}_{i \in I}$ to be a covering family if $\bigcup_{i \in I} f_{i}\left(A_{i}\right)=A$. Then $K_{0}($ FinSet $) \cong \mathbb{Z}$, with the set $\{1, \ldots, n\}$ representing $n \in \mathbb{Z}$.

If $k$ is a finite field then the functor $X \longmapsto X(k)$ gives a morphism of assemblers $\mathcal{V} \longrightarrow$ FinSet, and thus a map of spectra $K(\mathcal{V}) \longrightarrow K($ FinSet $)$. On $K_{0}$ this is just point counting over $k$. By 
the Barratt-Priddy-Quillen Theorem, $K($ FinSet $) \simeq \mathbb{S}$, the sphere spectrum, so we see that point counting lifts to a map of spectra $K(\mathcal{V}) \longrightarrow \mathbb{S}$.

There are two other important types of assemblers that arise in our analysis of $K(\mathcal{V})$.

Example 1.5. Let $G$ be a discrete group. The assembler $\mathbb{S}_{G}$ has two objects $\varnothing$ and $*$, with one morphism $\varnothing \longrightarrow *$ and $\operatorname{Aut}(*) \cong G$. Then $K\left(\mathbb{S}_{G}\right) \simeq \Sigma_{+}^{\infty} B G$; in particular, when $G$ is trivial $\mathbb{S}_{G}$ is weakly equivalent to the sphere spectrum.

Example 1.6. Suppose that $\mathcal{C}$ and $\mathcal{D}$ are assemblers. Then we can construct an assembler $\mathcal{C} \vee \mathcal{D}$ whose underlying category is the union of the categories $\mathcal{C}$ and $\mathcal{D}$ with the two initial objects glued together. The Grothendieck topology on $\mathcal{C} \vee \mathcal{D}$ is inherited from the topologies on $\mathcal{C}$ and $\mathcal{D}$.

The assembler $\mathcal{V}$ comes with an associated filtration induced by the dimension of the corresponding varieties. More concretely, let $\mathcal{V}^{(n)}$ be the full subcategory of varieties of dimension at most $n$; it has the structure of an assembler induced from that of $\mathcal{V}$, and the inclusion is a morphism of assemblers. Thus we get a diagram

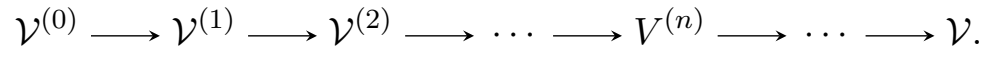

Applying $K$ to this sequence, we get a sequence of morphisms of spectra

$$
K\left(\mathcal{V}^{(0)}\right) \longrightarrow K\left(\mathcal{V}^{(1)}\right) \longrightarrow K\left(\mathcal{V}^{(2)}\right) \longrightarrow \cdots \longrightarrow K\left(V^{(n)}\right) \longrightarrow \cdots \longrightarrow K(\mathcal{V}),
$$

such that $K(\mathcal{V})$ is the colimit of this diagram; thus $K(\mathcal{V})$ is a filtered spectrum.

Remark 1.7. In order for some of the later techniques used in the paper to work, we need the morphisms of spectra to be cofibrations in some stable model structure on $\mathbf{S p}$. In order to accomplish this, we take as our model of the stable homotopy category the category of symmetric spectra of simplicial sets with the stable model structure. (See [MMSS01, Theorem9.2].) In this structure, the weak equivalences are the stable equivalences and the cofibrations are the level cofibrations. The construction of $K$ defined in [ZakA, Definition 1.9] gives the desired properties.

We now want to compute the spectral sequence induced by this filtered spectrum. In order to do this, we must first compute $\pi_{q}\left(K(\mathcal{V})^{(p)}, K(\mathcal{V})^{(p-1)}\right)$ for all $q$ and $p$. We will not give the full details of the proof; instead, we explain how to use the machinery developed in [ZakA] to obtain these results.

We write $\mathcal{V}^{(n, n-1)}$ for the assembler whose underlying category is the full subcategory of $\mathcal{V}$ consisting of varieties of dimension exactly $n$ and the empty variety. For a variety $X$, a family $\left\{f_{i}: X_{i} \longrightarrow X\right\}$ is a covering family if it can be completed to a disjoint covering family inside $\mathcal{V}^{(n)}$ by morphisms whose domains have dimension at most $n-1$. Thus for an irreducible variety $X$, a family $\left\{f_{i}: X_{i} \longrightarrow X\right\}_{i \in I}$ is a covering family if and only if $X_{i} \neq \varnothing$ for a unique $i \in I$.

Theorem 1.8. The cofiber of the map $K\left(\mathcal{V}^{(n-1)}\right) \hookrightarrow K\left(\mathcal{V}^{(n)}\right)$ is $K\left(\mathcal{V}^{(n, n-1)}\right)$.

This is a special case of [ZakA, Theorem D]. Computing the homotopy type of $K\left(\mathcal{V}^{(n, n-1)}\right)$ require the use of an approximation theorem for assemblers:

Theorem 1.9 ([ZakA, Theorem B]). Suppose that $\mathcal{D}$ is a subassembler of $\mathcal{C}$ such that every object $A$ of $\mathcal{C}$ has a finite disjoint covering family $\left\{f_{i}: A_{i} \longrightarrow A\right\}_{i \in I}$ such that $A_{i}$ is in $\mathcal{D}$ for all $i$. Then the map $K(\mathcal{D}) \longrightarrow K(\mathcal{C})$ induced by the inclusion $\mathcal{D} \hookrightarrow \mathcal{C}$ is a homotopy equivalence of spectra.

This is a devissage result for assemblers: if the objects in an assembler can be covered by the objects in a subassembler, then the inclusion of assemblers induces a homotopy equivalence on $K$-theory. For example, let $\mathcal{V}^{s m}$ be the full subcategory of $\mathcal{V}$ consisting of the smooth varieties. Then the inclusion $\mathcal{V}^{s m} \longrightarrow \mathcal{V}$ is an inclusion that satisfies the conditions of the proposition, since any variety can be stratified by smooth varieties, and thus the inclusion $K\left(\mathcal{V}^{s m}\right) \longrightarrow K(\mathcal{V})$ is a homotopy equivalence of spectra. 
Definition 1.10. Let $B_{n}$ be the set of birational isomorphism classes of irreducible varieties over $k$ of dimension $n$. For any $\alpha$ in $B_{n}$, define

$$
\operatorname{Aut}(\alpha)=\operatorname{Aut}_{k} k(X)
$$

for any variety $X$ in the birational isomorphism class $\alpha$.

Theorem $\mathrm{A}$ follows directly from several results in [ZakA]. Here, we give an outline of the proof by reducing of the theorem to those results.

Proof of Theorem A. By Theorem 1.8, it suffices to show that for any $n \geq 0$,

$$
K\left(\mathcal{V}^{(n, n-1)}\right) \simeq \bigvee_{\alpha \in B_{n}} \Sigma_{+}^{\infty} B \operatorname{Aut}(\alpha)
$$

Let $\widetilde{\mathcal{V}}^{(n, n-1)}$ be the full subassembler of $\mathcal{V}^{(n, n-1)}$ of irreducible varieties. By Theorem 1.9,

$$
K\left(\widetilde{\mathcal{V}}^{(n, n-1)}\right) \simeq K\left(\mathcal{V}^{(n, n-1)}\right)
$$

Pick a representative $X_{\alpha}$ for each equivalence class $\alpha$ in $B_{n}$, and let $\mathcal{C}$ be the full subassembler of $\widetilde{\mathcal{V}}^{(n, n-1)}$ consisting of subvarieties of $X_{\alpha}$ for any $\alpha$. By Theorem 1.9, $K(\mathcal{C}) \simeq K\left(\mathcal{V}^{(n, n-1)}\right)$. Note that each nonempty variety in $\mathcal{C}$ has a morphism to exactly one of the $X_{\alpha}$, since all morphisms are inclusions of dense open subsets and if some $Z$ had morphisms to $X_{\alpha}$ and $X_{\beta}$ then $X_{\alpha}$ and $X_{\beta}$ are by definition birationally isomorphic, hence $\alpha=\beta$. We can therefore write

$$
\mathcal{C} \cong \bigvee_{\alpha \in B_{n}} \mathcal{C}_{X_{\alpha}}
$$

where $\mathcal{C}_{X_{\alpha}}$ is the full subassembler of $\mathcal{C}$ consisting of those varieties with morphisms to $X_{\alpha}$. $K$ theory commutes with $\vee$, so it remains to analyze $K\left(\mathcal{C}_{X_{\alpha}}\right)$ for all $\alpha$ in $B_{n}$. This is done in [ZakA, Theorem 2.1(1)], which states exactly that $K\left(\mathcal{C}_{X_{\alpha}}\right)=\Sigma_{+}^{\infty} B \operatorname{Aut}\left(X_{\alpha}\right)$.

We also need a construction of the cofiber of a morphism of (simplicial) assemblers, by which we mean functors $\Delta^{o p} \longrightarrow$ Asm. In general, the cofiber of a morphism of assemblers (or a morphism of simplicial assemblers) is a simplicial assembler, so this level of generality is necessary. The category of assemblers sits inside the category of simplicial assemblers as the constant simplicial assemblers. (For more on simplicial objects, see for example [Hov99, Chapter 3]; for a more in-depth discussion of this definition and Theorem 1.12 see [ZakA, Section 4].)

Definition 1.11. For any assembler, $\mathcal{C}$, write $\nabla: \mathcal{C} \vee \mathcal{C} \longrightarrow \mathcal{C}$ for the fold map which is given by the identity on each of the two wedge summands.

Let $F: \mathcal{C}$. $\longrightarrow \mathcal{D}$. be a morphism of simplicial assemblers. The simplicial assembler $(\mathcal{D} . / F)$. is defined by

$$
(\mathcal{D} . / F)_{n}=\mathcal{D}_{n} \vee \bigvee_{i=1}^{n} \mathcal{C}_{n}
$$

The face maps $d_{i}$ for $n>i>0$ are defined by the composition

$$
\mathcal{C}_{n} \vee \mathcal{C}_{n} \stackrel{d_{i} \vee d_{i}}{\longrightarrow} \mathcal{C}_{n-1} \vee \mathcal{C}_{n-1} \stackrel{\nabla}{\longrightarrow} \mathcal{C}_{n-1}
$$

on the $i$-th and $i+1$-st copies of $\mathcal{C}_{n}$, and by $d_{i}$ on the other wedge summands. The map $d_{0}$ is defined by the composition

$$
\mathcal{D}_{n} \vee C_{n} \stackrel{d_{0} \vee d_{0}}{\longrightarrow} \mathcal{D}_{n-1} \vee \mathcal{C}_{n-1} \stackrel{1 \vee F}{\longrightarrow} \mathcal{D}_{n-1} \vee \mathcal{D}_{n-1} \stackrel{\nabla}{\longrightarrow} \mathcal{D}_{n-1}
$$

on the first two wedge summands and $d_{0}$ on the others, and $d_{n}$ is defined by mapping the $n$-th copy of $\mathcal{C}_{n}$ entirely to the initial object and by $d_{n}$ elsewhere.

By abusing notation, we generally write $\mathcal{D} . / F$ for the simplicial assembler $(\mathcal{D} . / F)$; when $\mathcal{D}$. is a constant assembler $\mathcal{D}$ we write $\mathcal{D} / F$. 
There is a morphism of simplicial assemblers $p: \mathcal{D} . \longrightarrow(\mathcal{D} / F)$. given by including $\mathcal{D}_{n}$ into the $\mathcal{D}_{n}$ in the $n$-th level.

In the case when $\mathcal{D}$. is the assembler which is trivial at each level, we write $\Sigma \mathcal{C}$. for $\mathcal{D} . / F$.

This construction is analogous to the usual bar construction for a cofiber of a map of simplicial objects; for more on bar constructions, see [Rie14, Section 4.2].

Theorem 1.12 ([ZakA, Theorem $\mathrm{C}])$. The sequence

$$
K(\mathcal{C} .) \stackrel{K(F)}{\longrightarrow} K(\mathcal{D} .) \stackrel{K(p)}{\longrightarrow} K((\mathcal{D} / F) .)
$$

is a cofiber sequence. The boundary morphism $K_{n}((\mathcal{D} / F)$. $) \longrightarrow K_{n-1}(\mathcal{C}$. $)$ is obtained from the morphism of simplicial assemblers $(\mathcal{D} / F) . \longrightarrow \Sigma \mathcal{C}$. defined by sending each copy of $\mathcal{D}_{n}$ to the initial object.

\section{AN ASIDE ON SPECTRAL SEQUENCES}

This section contains a quick introduction to the construction of the spectral sequence associated to a filtered spectrum, geared towards the results we need in later sections. In order to make our results easier to read we use a nonstandard grading in the spectral sequences. All of the material in this section is well-known; for a reference, see [McC01].

Suppose that we have a filtered spectrum, by which we mean a diagram

$$
X_{0} \stackrel{i_{1}}{\longrightarrow} X_{1} \stackrel{i_{2}}{\longrightarrow} \cdots \stackrel{i_{n}}{\longrightarrow} X_{n} \stackrel{i_{n+1}}{\longrightarrow} \cdots \longrightarrow X,
$$

where $X=\operatorname{colim}_{n} X_{n}$. Then for each $n$ we have a long exact sequence

$$
\cdots \longrightarrow \pi_{m}\left(X_{n-1}\right) \stackrel{\iota}{\longrightarrow} \pi_{m}\left(X_{n}\right) \stackrel{p}{\longrightarrow} \pi_{m}\left(X_{n}, X_{n-1}\right) \stackrel{\partial}{\longrightarrow} \pi_{m-1}\left(X_{n-1}\right) \longrightarrow \cdots
$$

Since $X$ is a spectrum, this sequence is infinite in both directions. We define

$$
A_{p, q}=\pi_{p}\left(X_{q}\right) \quad \text { and } \quad E_{p, q}^{1}=\pi_{p}\left(X_{q}, X_{q-1}\right) ;
$$

from the long exact sequences we then have the following diagram:

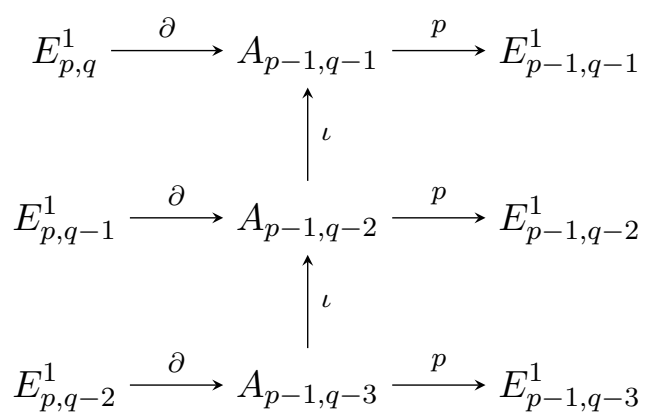

By gluing copies of itself for different $p$ and $q$ this diagram can be continued infinitely in all directions. Note that each group $E_{p, q}^{1}$ and $A_{p, q}$ appears in it only once, and that the original long exact sequences appear as stair-steps: for example, the sequence

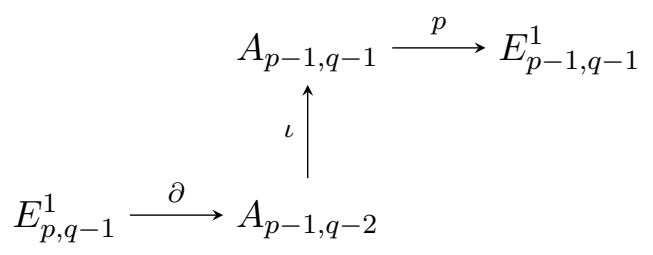


is exact.

The differential $d_{1}: E_{p, q}^{1} \longrightarrow E_{p-1, q-1}^{1}$ is defined to be $p \partial$ and $E_{p, q}^{2} \stackrel{\text { def }}{=} \operatorname{ker} d_{1} /$ im $d_{1}$. If $a$ is in ker $d_{1}$ then $\partial a$ must be in $\operatorname{ker} p=\operatorname{im} \iota$; thus there exists $a^{\prime}$ in $A_{p-1, q-2}$ such that $\iota\left(a^{\prime}\right)=a$. We define $d_{2}(a) \stackrel{\text { def }}{=} p\left(a^{\prime}\right)$, and $E_{p, q}^{3} \stackrel{\text { def }}{=} \operatorname{ker} d_{2} / \operatorname{im~} d_{2}$. This continues onwards: if $d_{2}(a)=0$ then $a^{\prime}$ is in $\operatorname{ker} p=\operatorname{im} \iota$ and thus there exists $a^{\prime \prime}$ in $A_{p-1, q-3}$ such that $\iota\left(a^{\prime \prime}\right)=a^{\prime}$; we define $d_{3}(a) \stackrel{\text { def }}{=} p\left(a^{\prime \prime}\right)$, and so on. In general,

$$
d_{r}: E_{p, q}^{r} \longrightarrow E_{p-1, q-r}^{r} .
$$

We note the following lemma as it is useful later:

Lemma 2.1. For $x$ in $E_{p, q}^{1}$, if $\partial x=0$ then $d_{r} x=0$ for all $r \geq 1$.

With this indexing we have a spectral sequence

$$
E_{p, q}^{1} \Longrightarrow \pi_{p}(X)
$$

in which $d_{r}: E_{p, q}^{r} \longrightarrow E_{p-1, q-r}^{r}$. If we write $I_{n}: X_{n} \longrightarrow X$ then on the $E^{\infty}$ page we have

$$
E_{p, q}^{\infty}=\left(I_{q}\right)_{*} \pi_{p}\left(X_{q}\right) /\left(\left(I_{q-1}\right)_{*} \pi_{p}\left(X_{q-1}\right)\right) .
$$

Thus the $p$-th column of the $E^{\infty}$ page is the associated graded of the filtration on $\pi_{p}(X)$ induced by the filtered spectrum. As a consequence of [Boa99, Theorem 6.1] we get the following:

Lemma 2.3. If all of the spectra $X_{i}$ are connective (all $\pi_{i}=0$ for $i<0$ ) then this spectral sequence converges to the homotopy groups of $X$.

\section{A speCtral SEQUenCE FOR $K(\mathcal{V})$}

The dimension filtration on $\mathcal{V}$ produces a filtered spectrum

$$
K\left(\mathcal{V}^{(0)}\right) \longleftrightarrow K\left(\mathcal{V}^{(1)}\right) \longleftrightarrow \cdots \hookrightarrow K(\mathcal{V}) .
$$

We now use the tools of Section 2 to write down and analyze the spectral sequence we get from this filtered spectrum.

The filtration on $K(\mathcal{V})$ gives us the following spectral sequence:

$$
E_{p, q}^{1}=K_{p}\left(\mathcal{V}^{(q, q-1)}\right) \Longrightarrow K_{p}(\mathcal{V}) \text {. }
$$

The differential $d_{r}$ is a homomorphism $E_{p, q}^{r} \longrightarrow E_{p-1, q-r}^{r}$. As $E_{q-r}^{r}$ is 0 for all $r>q$ Lemma 2.3 applies and the spectral sequence converges. We know that

$$
\pi_{0}\left(\Sigma_{+}^{\infty} B G\right) \cong \mathbb{Z} \quad \text { and } \quad \pi_{1}\left(\Sigma_{+}^{\infty} B G\right) \cong G^{\mathrm{ab}} \oplus \mathbb{Z} / 2,
$$

and since $\pi_{*}$ is a homology theory applying $\pi_{*}$ changes $\bigvee$ to $\bigoplus$. Therefore we can compute the first two columns of the spectral sequence; a picture of this appears in Figure 1 .

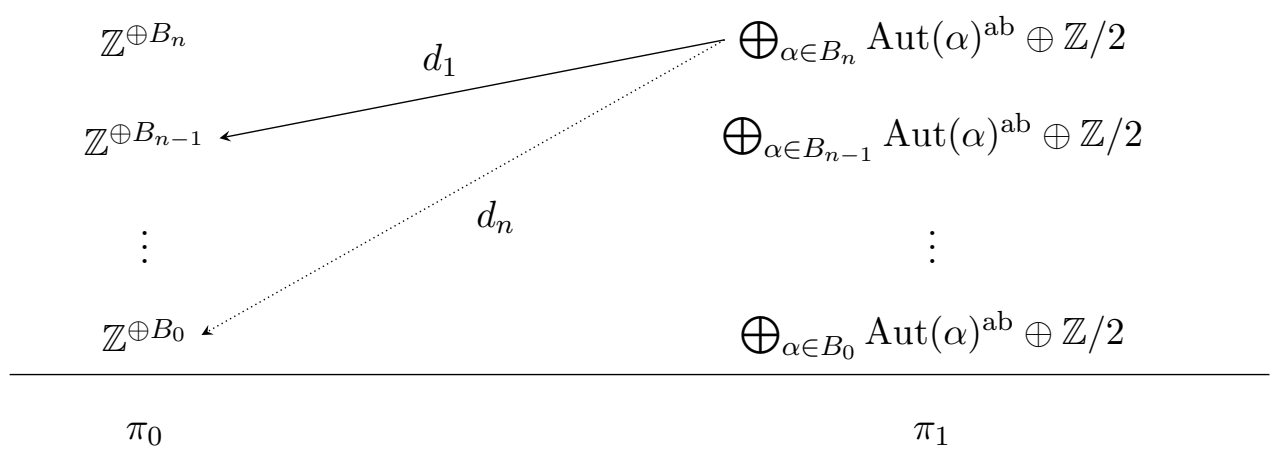

FiguRE 1. Spectral sequence for $K(\mathcal{V})$ 
We would like to compute the differentials in this spectral sequence. To do this, we first compute the boundary map $\partial: K_{1}\left(\mathcal{V}^{(q, q-1)}\right) \longrightarrow K_{0}\left(\mathcal{V}^{(q-1)}\right)$ in the long exact sequence for the cofiber sequence $K\left(\mathcal{V}^{(q-1)}\right) \longrightarrow K\left(\mathcal{V}^{(q)}\right) \longrightarrow K\left(\mathcal{V}^{(q, q-1)}\right)$.

Lemma 3.2. Let $\alpha$ be in $B_{q}$ and let $\varphi$ be in $\operatorname{Aut}(\alpha)$. For a representative $X$ of $\alpha$, let $\varphi$ be represented by an isomorphism $U \longrightarrow V$ of dense open subsets of $X$. Then

$$
\partial[\varphi]=[X \backslash V]-[X \backslash U] .
$$

Proof. From [ZakB, Corollary 3.10] we know that each $x$ in $K_{1}\left(\mathcal{V}^{(q, q-1)}\right)$ can be represented by elements consisting of the following data: two finite disjoint covering families

$$
\left\{f_{i}: X_{i} \longrightarrow X\right\}_{i \in I} \cup\{f: Y \longrightarrow X\}
$$

and

$$
\left\{g_{i}: X_{i} \longrightarrow X\right\}_{i \in I} \cup\left\{g^{\prime}: Z \longrightarrow X\right\}
$$

such that $\operatorname{dim} Y, \operatorname{dim} Z \leq q-1$. More informally, an element of $K_{1}\left(\mathcal{V}^{(q, q-1)}\right)$ consists of a variety $X$, a dense open subset (represented by the union of the $X_{i}$ 's) which is embedded into $X$ in two different ways (represented by the maps $f_{i}$ and $g_{i}$ ) as well as the data of the two different complements to the embeddings in $X$ ( $Y$ and $Z)$. Applying [ZakB, Proposition 3.13] to this representation we get that

$$
\partial x=[Z]-[Y]
$$

in $K_{0}\left(\mathcal{V}^{(q-1)}\right)$.

Now consider $\varphi$. The class of $\varphi$ in $K\left(\mathcal{V}^{(q, q-1)}\right)$ can be represented by the two covering families

$$
\{U \longrightarrow X\} \cup\{X \backslash U \longrightarrow X\} \text { and }\{U \stackrel{\varphi}{\longrightarrow} V \longrightarrow X\} \cup\{X \backslash V \longrightarrow X\} .
$$

Therefore $\partial[\varphi]=[X \backslash V]-[X \backslash U]$.

Thus the boundary map in the long exact sequence associated to the inclusion of one filtration degree into the next measures the error of a birational automorphism of the variety extending to a piecewise automorphism. In order to make this connection more precise, we start with the following lemma.

Lemma 3.3. Let $X$ and $Y$ be varieties of dimension at most $n$ such that $[X]=[Y]$ in $K_{0}\left(\mathcal{V}^{(n, n-1)}\right)$. Then we can write $X=U \uplus X^{\prime}$ and $Y=V \uplus Y^{\prime}$ such that $U$ and $V$ are piecewise isomorphic and $\operatorname{dim} X^{\prime}, \operatorname{dim} Y^{\prime}<n$.

Proof. Write $X=\bigcup_{i=0}^{n} X_{i}$, where for $i>0$ the $X_{i}$ are the irreducible components of $X$ of dimension $n$, and $X_{0}$ is the union of all of the irreducible components of $X$ which have dimension less than $n$. For $i>0$, let $U_{i}=X_{i} \backslash \bigcup_{j \neq i} X_{j}$, and let $X_{0}^{\prime}=X \backslash \bigcup_{i=1}^{n} U_{i}$. Thus we have $X=X_{0}^{\prime} \uplus \biguplus_{i=1}^{n} U_{i}$ where $\operatorname{dim} X_{0}^{\prime}<n, \operatorname{dim} U_{i}=n$ and each $U_{i}$ is irreducible. Analogously, $Y$ can be written as $Y_{0}^{\prime} \uplus \biguplus_{j=1}^{m} V_{j}$.

Since all varieties of dimension less than $n$ are 0 in $K_{0}\left(\mathcal{V}^{(n, n-1)}\right),[X]=\sum_{i=1}^{n}\left[U_{i}\right]$ and $[Y]=$ $\sum_{j=1}^{m}\left[V_{j}\right]$. Since every open subset of an irreducible variety is dense and $[X]=[Y]$, it follows that $m=n$, and we must have a permutation $\sigma$ in $\Sigma_{n}$ such that $\left[U_{i}\right]=\left[V_{\sigma(i)}\right]$ in $K_{0}\left(\mathcal{V}^{(n, n-1)}\right)$. Thus for each $i \geq 1$ there exists a birational isomorphism $U_{i} \rightarrow V_{\sigma(i)}$, given as an isomorphism $\varphi_{i}: U_{i}^{\prime} \longrightarrow V_{\sigma(i)}^{\prime}$. Let $X_{i}^{\prime}=U_{i} \backslash U_{i}^{\prime}$ and $Y_{i}^{\prime}=V_{i} \backslash V_{i}^{\prime}$; note that $\operatorname{dim} X_{i}^{\prime}, \operatorname{dim} Y_{i}^{\prime}<n$. Thus if we define $X^{\prime}=\biguplus_{i=0}^{n} X_{i}^{\prime}$ and $U=\biguplus_{i=1}^{n} U_{i}^{\prime}$ then $X=X^{\prime} \uplus U$ and $\operatorname{dim} X^{\prime}<n$. Similarly, we can define $Y^{\prime}=\biguplus_{i=0}^{n} Y_{i}^{\prime}$ and $V=\biguplus_{i=1}^{n} V_{i}^{\prime}$. By definition $U$ and $V$ are piecewise isomorphic and we are done.

The next theorem shows that the spectral sequence constructs an obstruction to $\varphi$ extending to a piecewise isomorphism, thereby answering the question of Gromov in [Gro99, p121]. It also directly implies Theorem B. 
Theorem 3.4. A birational automorphism $\varphi$ of an irreducible variety $X$ extends to a piecewise automorphism of $X$ if and only if $d_{r}[\varphi]=0$ for all $r \geq 1$.

Proof. Suppose that $\operatorname{dim} X=q$. Since $X$ is irreducible it is represented by a class in $B_{q}$, and thus participates in the spectral sequence. Note that in the rest of the proof all unions are disjoint; however, since we are not taking coproducts of varieties but rather thinking of a decomposition of a given variety, we use the symbol $\uplus$ rather than $\amalg$. Suppose that $\varphi$ is defined as an isomorphism $U \longrightarrow V$ for $U \subseteq X$ and $V \subseteq Y$.

First, suppose that $\varphi$ extends to a piecewise automorphism. Then $[X \backslash V]=[X \backslash U]$ in $K_{0}\left[\mathcal{V}^{(q-1)}\right]$ and thus $\partial[\varphi]=0$. Then by Lemma $\left[2.1, d_{r}[\varphi]=0\right.$ for all $r \geq 1$.

Now suppose that $d_{r}[\varphi]=0$ for all $r \geq 1$. Since $d_{1}[\varphi]=0$ it follows that $[X \backslash U]=[X \backslash V]$ in $K_{0}\left(\mathcal{V}^{(q, q-1)}\right)$. We show by induction that we can write $X=U_{r} \uplus X_{r}^{\prime}=V_{r} \uplus Y_{r}^{\prime}$ with $U_{r}$ and $V_{r}$ piecewise isomorphic, $\operatorname{dim} X_{r}^{\prime}, \operatorname{dim} Y_{r}^{\prime}<n-r$ and $\partial[\varphi]=\left[Y_{r}^{\prime}\right]-\left[X_{r}^{\prime}\right]$. Then setting $r=n$ gives us the desired result.

The base case $r=0$ is given to us by the definition of $\varphi$ : define $U_{0}=U$ and $V_{0}=V$, and $X_{0}^{\prime}=X \backslash U_{0}, Y_{0}^{\prime}=X \backslash V_{0}$. We assume the case for $r-1$, and prove it for $r$. By the inductive hypothesis $\partial[\varphi]=\left[Y_{r-1}^{\prime}\right]-\left[X_{r-1}^{\prime}\right]$ we have

$$
d_{r}[\varphi]=\left[Y_{r-1}^{\prime}\right]-\left[X_{r-1}^{\prime}\right]
$$

in $K_{0}\left(\mathcal{V}^{(n-r, n-r-1)}\right)$. Since $d_{r}[\varphi]=0$ by assumption, Lemma 3.3 applies and we can write $X_{r-1}^{\prime}=$ $U_{r}^{\prime} \uplus X_{r}^{\prime}$ and $Y_{r-1}^{\prime}=V_{r}^{\prime} \uplus Y_{r}^{\prime}$ with $U_{r}^{\prime} \cong V_{r}^{\prime}$ and $\operatorname{dim} X_{r}^{\prime}, \operatorname{dim} Y_{r}^{\prime}<n-r$. If we set $U_{r}=U_{r-1} \uplus U_{r}^{\prime}$ and $V_{r}=V_{r-1} \uplus V_{r}^{\prime}$ then $U_{r}$ and $V_{r}$ are piecewise isomorphic. Thus all that we need to check to finish the induction is the formula for $\partial[\varphi]$. This is straightforward, as

$$
\partial[\varphi]=\left[Y_{r-1}^{\prime}\right]-\left[X_{r-1}^{\prime}\right]=\left[V_{r}^{\prime}\right]+\left[Y_{r}^{\prime}\right]-\left[U_{r}^{\prime}\right]-\left[X_{r}^{\prime}\right]=\left[Y_{r}^{\prime}\right]-\left[X_{r}^{\prime}\right],
$$

since $U_{r}^{\prime}$ and $V_{r}^{\prime}$ are isomorphic.

Remark 3.5. In Figure 1 each summand in the $\pi_{1}$-column has a $\mathbb{Z} / 2$ component, which we ignored in the above discussion. This is because $d_{r}$ is uniformly zero on all of these. To see this, note that we have morphisms of assemblers

$$
\mathbb{S} \longrightarrow \mathbb{S}_{G} \longrightarrow \mathbb{S}
$$

which take $\varnothing$ to $\varnothing$ and $*$ to $*$. The composition of these morphisms is the identity. We know that $K\left(\mathbb{S}_{G}\right) \simeq \Sigma_{+}^{\infty} B G \simeq \mathbb{S} \vee \Sigma^{\infty} B G$, with the $\mathbb{S}$ coming from the $K(\mathbb{S})$ that is split off by the above sequence. This $\mathbb{S}$ keeps track of the combinatorial details of what is going on: its $\pi_{0}$ is $\mathbb{Z}$ and corresponds to the number of varieties we are considering, and $\pi_{1}$ keeps track of the permutations of the varieties (and corresponds to the sign of the permutation.) The $\mathbb{Z} / 2$ in each component of $\pi_{1}$ comes from the $\mathbb{S}$ summand, and using this we write down representatives for the $\mathbb{Z} / 2$ indexed by $\alpha$ : it is represented by the two families

$$
\{X \sqcup X \stackrel{\tau}{\longrightarrow} X \sqcup X\} \cup\{\} \quad \text { and } \quad\{X \sqcup X \stackrel{=}{\longrightarrow} X \sqcup X\} \cup\{\}
$$

where $[X]=\alpha$ in $B_{q}$. Thus $\partial$ on this generator is 0 .

\section{Multiplication By $\mathbb{L}$}

Now consider the morphism of assemblers $L: \mathcal{V} \longrightarrow \mathcal{V}$ which sends the variety $X$ to $X \times \mathbb{A}^{1}$, and let $C$ be the cofiber of the map $K(L)$ of spectra. Then we have a long exact sequence in homotopy given by

$$
K_{1}(\mathcal{V}) \stackrel{p_{1}}{\longrightarrow} \pi_{1} C \longrightarrow K_{0}(\mathcal{V}) \stackrel{\cdot \mathbb{L}}{\longrightarrow} K_{0}(\mathcal{V}) \longrightarrow \pi_{0} C \longrightarrow 0
$$

The cokernel of $p_{1}$ is equal to the kernel of multiplication by $\mathbb{L}$. Thus $\mathbb{L}$ is a zero divisor if and only if $p_{1}$ is a surjection. 
We thus want to analyze the homotopy type of $C$ together with the image of $p_{1}$. It turns out that it is possible to construct $C$ as the $K$-theory of a simplicial assembler and to analyze this map through the structure of assemblers. Applying Theorem 1.12, we can write $C=K(\mathcal{V} / L)$. By the construction in Theorem 1.12, $\mathcal{V}^{(n)} / L$ is a simplicial subassembler of $\mathcal{V}^{(n+1)} / L$, and (as mentioned in Remark 1.7) the inclusion $\mathcal{V}^{(n)} / L \hookrightarrow \mathcal{V}^{(n+1)} / L$ gives a cofibration on $K$-theory. We get the following commutative diagram:

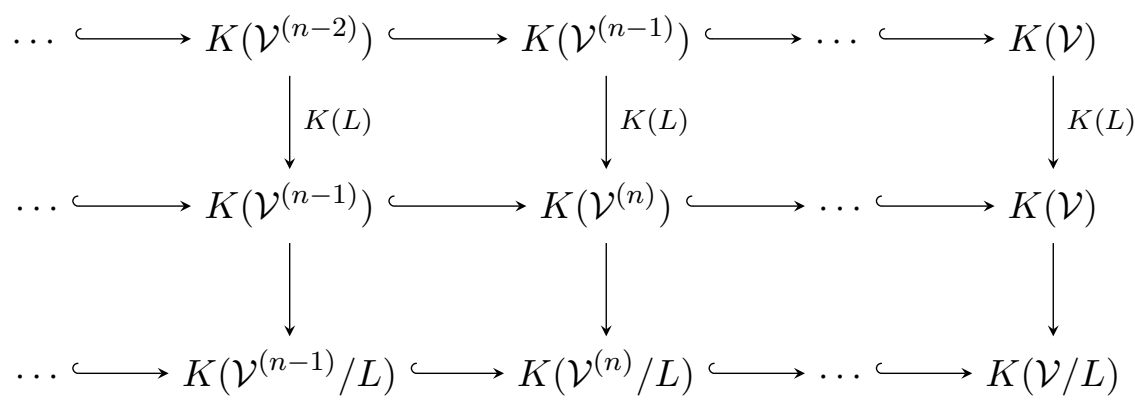

We define the function

$$
\ell: B_{n-1} \longrightarrow B_{n} \quad \text { by } \quad \ell[X]=\left[X \times \mathbb{A}^{1}\right] .
$$

Proposition 4.3. For $\beta$ in $B_{n}$, let $\nabla_{\beta}: \bigvee_{\ell^{-1}(\beta)} \mathbb{S} \longrightarrow \mathbb{S}$ be the fold map, and let

$$
C_{\beta}=\operatorname{cofib} \nabla_{\beta} \vee \operatorname{cofib}\left(\bigvee_{\alpha \in \ell^{-1}(\beta)} \Sigma^{\infty} B \operatorname{Aut}(\alpha) \longrightarrow \Sigma^{\infty} B \operatorname{Aut}(\beta)\right) .
$$

There exists a spectral sequence

$$
\widetilde{E}_{p, q}^{1}=\bigoplus_{\beta \in B_{q}} \pi_{p} C_{\beta} \Longrightarrow \pi_{p} K(\mathcal{V} / L)
$$

Proof. It suffices to show that

$$
\operatorname{cofib}\left(K\left(\mathcal{V}^{(n-1)} / L\right) \rightarrow K\left(\mathcal{V}^{(n)} / L\right)\right) \simeq \bigvee_{\beta \in B_{n}} C_{\beta}
$$

Then the spectral sequence from (2.2) for the filtration on $K(\mathcal{V} / L)$ is the one in the statement of the proposition. Therefore we focus on proving the claim. For this proof, we restrict our attention to the subassembler of $\mathcal{V}$ consisting of only irreducible varieties; in order to avoid cluttering the notation, we still denote it by $\mathcal{V}$. By Theorem 1.9 this gives the correct homotopy type.

Write $\iota: \mathcal{V}^{(n-1)} \hookrightarrow \mathcal{V}^{(n)}$, and $\widetilde{\iota:} V^{(n-1)} / L \longrightarrow \mathcal{V}^{(n)} / L$. Then we have a diagram

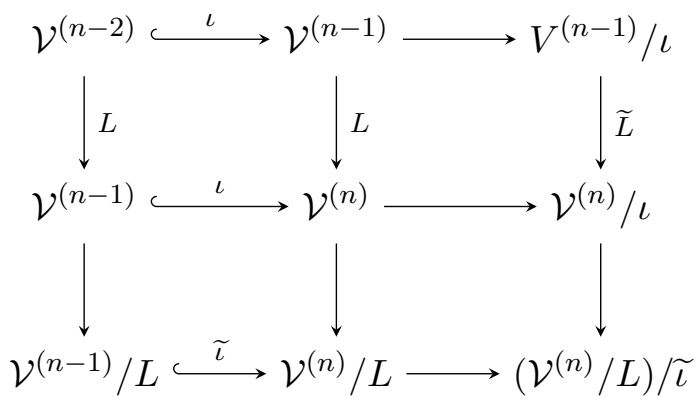


From Definition 1.11 we have $\left(\mathcal{V}^{(n)} / L\right) / \widetilde{\iota} \cong\left(\mathcal{V}^{(n)} / \iota\right) / \widetilde{L}$. Therefore if we want to determine the homotopy type of $K\left(\left(\mathcal{V}^{(n)} / L\right) / \widetilde{\iota}\right)$ it suffices to determine the cofiber of the map

$$
K(\widetilde{L}): K\left(\mathcal{V}^{(n-1)} / \iota\right) \longrightarrow K\left(\mathcal{V}^{(n)} / \iota\right) .
$$

We have a morphism of assemblers $V^{(n)} / \iota \longrightarrow \mathcal{V}^{(n, n-1)}$ given by mapping a variety of dimension $n$ to itself and a variety of dimension less than $n$ to the initial object. Then the diagram

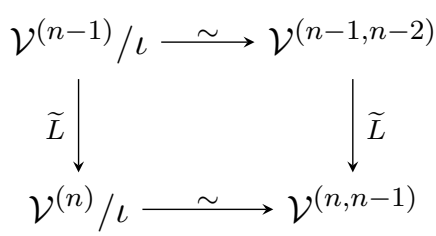

commutes, and by Theorem 1.8 the two horizontal morphisms are weak equivalences after applying $K$-theory. Thus after applying $K$-theory, the cofiber of the left-hand map $\widetilde{L}$ is the same as the cofiber of the right-hand map $\widetilde{L}$.

For $\beta$ in $B_{n}$, let $\left.\left(\mathcal{V}^{(n, n-1)}\right)\right|_{\beta}$ be the full subassembler of those varieties whose birational isomorphism class is $\beta$. Then we can rewrite the right-hand column as

$$
\bigvee_{\beta \in B_{n}}\left(\left.\left(\left.\bigvee_{\alpha \in \ell^{-1}(\beta)} \mathcal{V}^{(n-1, n-2)}\right|_{\alpha}\right) \longrightarrow \mathcal{V}^{(n, n-1)}\right|_{\beta}\right) .
$$

Since $K$-theory and cofibers commute with coproducts, it suffices to show that the cofiber of each term in the above wedge product is weakly equivalent to $C_{\beta}$. We want to show that the splitting from Theorem $\mathrm{A}$ is compatible with this map, so that we can compute cofibers in terms of homomorphisms of groups instead of in terms of maps of spaces.

We therefore aim to construct a splitting of both sides of the map simultaneously. Fix a representative $X_{\beta}$. For each $\alpha$ in $\ell^{-1}(\beta)$, choose an isomorphism $\psi_{\alpha}:$ Aut $_{k} k\left(X_{\alpha} \times \mathbb{A}^{1}\right) \longrightarrow \operatorname{Aut}_{k} k\left(X_{\beta}\right)$. For any variety $Y$ with a chosen embedding $Y \hookrightarrow \mathbb{A}^{N}$ we let $\mathcal{C}_{Y}$ be the assembler of subvarieties of $Y$, considered as algebraic subsets of $Y(\bar{k})$ defined over $k$. We then have the following diagram:

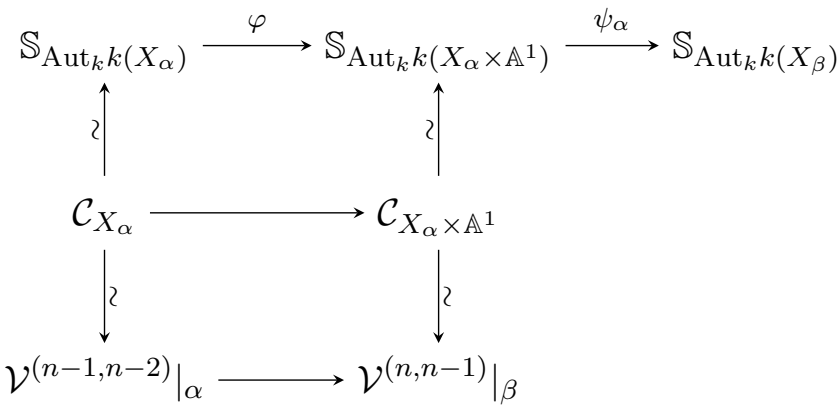

Note that the morphism across the bottom is a component of the map we're trying to find the cofiber of. The morphisms in the top row are all induced by group homomorphisms. The first is induced by the inclusion of $\operatorname{Aut}_{k} k\left(X_{\alpha}\right)$ into $\operatorname{Aut}_{k} k\left(X_{\alpha} \times \mathbb{A}^{1}\right)$. By [ZakA, Theorem 2.1(2)], the top square commutes. The vertical maps from the second row to the third row take each subvariety to itself; they are equivalences after applying $K$ by Theorem 1.9. The bottom square commutes by definition. 
From this diagram we can conclude that taking the cofiber of the bottom row is weakly equivalent to taking the cofiber of the top row, and we see that

$$
\text { cofib } \bigvee_{\alpha \in \ell^{-1}(\beta)} K\left(\mathcal{V}^{(n-1, n-2)} \mid \alpha\right) \longrightarrow K\left(\left.\mathcal{V}^{(n, n-1)}\right|_{\beta}\right) \simeq \operatorname{cofib} \bigvee_{\alpha \in \ell^{-1}(\beta)} K\left(\mathbb{S}_{\operatorname{Aut}(\alpha)}\right) \longrightarrow K\left(\mathbb{S}_{\operatorname{Aut}(\beta)}\right)
$$

We now compute this cofiber. We would like the cofiber to split further into a part that just comes from a homomorphism of groups, and a part that comes from a fold map of sphere spectra. Note that we have the following commutative diagram of assemblers:

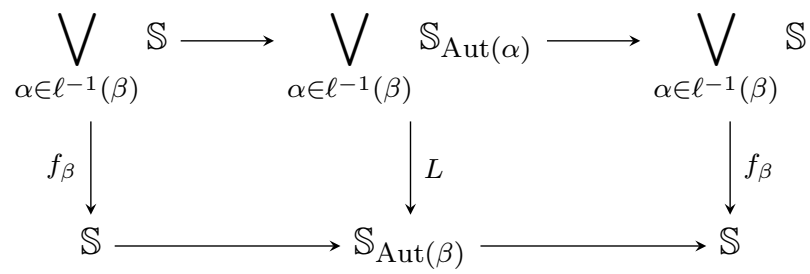

The maps $f_{\beta}$ are the fold maps, and $K\left(f_{\beta}\right) \simeq \nabla_{\beta}$. The horizontal morphisms are induced by the group homomorphisms $1 \longrightarrow G \longrightarrow 1$. Note that the compositions along the top and bottom rows are identity morphisms. Thus the cofiber of $K(L)$ splits as the wedge of the cofiber of $\nabla_{\beta}$ with the cofiber of

$$
\bigvee_{\alpha \in \ell^{-1}(\beta)}\left(\operatorname{cofib} K(\mathbb{S}) \longrightarrow K\left(\mathbb{S}_{\operatorname{Aut}(\alpha)}\right)\right) \longrightarrow\left(\operatorname{cofib} K(\mathbb{S}) \longrightarrow K\left(\mathbb{S}_{\operatorname{Aut}(\beta)}\right)\right)
$$

The desired statement follows from the observation that $\operatorname{cofib}\left(K(\mathbb{S}) \longrightarrow K\left(\mathbb{S}_{G}\right)\right) \simeq \Sigma^{\infty} B G$.

This is a much more complicated splitting result than the one we got in Theorem $\mathrm{A}$, but it turns out that we can still write down its $\pi_{0}$ and $\pi_{1}$. For simplicity, we write

$$
\widetilde{C}_{\beta} \stackrel{\text { def }}{=} \operatorname{cofib} \bigvee_{\alpha \in \ell^{-1}(\beta)} \Sigma^{\infty} B \operatorname{Aut}(\alpha) \longrightarrow \Sigma^{\infty} B \operatorname{Aut}(\beta),
$$

so that $C_{\beta}=\left(\operatorname{cofib} \nabla_{\beta}\right) \vee \widetilde{C}_{\beta}$.

First we compute $\pi_{i}\left(\right.$ cofib $\left.\nabla_{\beta}\right)$ for $i=0,1$. If $\ell^{-1}(\alpha)=\emptyset$, then cofib $\nabla_{\beta}=\mathbb{S}$, so $\pi_{0}=\mathbb{Z}$ and $\pi_{1}=\mathbb{Z} / 2$. On the other hand, if $\ell^{-1}(\alpha) \neq 0$, then $\pi_{0}\left(\nabla_{\beta}\right): \mathbb{Z}^{\oplus \ell^{-1}(\beta)} \longrightarrow \mathbb{Z}$ is addition, and in particular is surjective; therefore $\pi_{0}=0$. Thus we see that

$$
\pi_{0} \text { cofib } \nabla_{\beta} \cong\left\{\begin{array}{ll}
\mathbb{Z} & \text { if } \ell^{-1}(\beta)=\emptyset \\
0 & \text { otherwise. }
\end{array} .\right.
$$

Now consider $\pi_{1}$. The map $\nabla_{\beta}$ has a section, so in fact cofib $\nabla_{\beta} \simeq \bigvee_{\left|\ell^{-1}(\beta)\right|-1} \Sigma \mathbb{S}$. Of course, $\ell^{-1}(\beta)$ may be infinite, so the indexing on the sum doesn't quite make sense. However, we can still clearly identify what $\pi_{1}$ cofib $\nabla_{\beta}$ is: it is the subgroup of $\mathbb{Z}^{\oplus \ell^{-1}(\beta)}$ consisting of those formal sums $a_{1} \alpha_{1}+\cdots+a_{n} \alpha_{n}$ such that $a_{1}+\cdots+a_{n}=0$. Since this is a subgroup of "codimension" 1 the indexing is justified from that perspective. Thus in general, $\pi_{1}\left(\operatorname{cofib} \nabla_{\beta}\right) \cong \widetilde{\mathbb{Z}}^{\oplus \ell^{-1}(\beta)}$, justifying our use of this notation.

To compute $\pi_{0}$ and $\pi_{1}$ of $\widetilde{C}_{\beta}$ we use the long exact sequence in homotopy. Then we have a long exact sequence

$$
\bigoplus_{\alpha \in \ell^{-1}(\beta)} \operatorname{Aut}(\alpha)^{\mathrm{ab}} \stackrel{\ell}{\longrightarrow} \operatorname{Aut}(\beta)^{\mathrm{ab}} \longrightarrow \pi_{1} \widetilde{C}_{\beta} \longrightarrow 0 \longrightarrow 0 \longrightarrow \widetilde{C}_{\beta} \longrightarrow 0
$$


Thus we conclude that $\pi_{0} \widetilde{C}_{\beta}=0$ and

$$
\pi_{1} \widetilde{C}_{\beta}=\operatorname{Aut}(\beta)^{\mathrm{ab}} / \iota\left(\bigoplus_{\alpha \in \ell^{-1}(\beta)} \operatorname{Aut}(\alpha)^{\mathrm{ab}}\right)
$$

Note that even though $\operatorname{Aut}(\alpha) \longrightarrow \operatorname{Aut}(\beta)$ is only well-defined up to conjugation this formula is independent of those choices. Notice, also, that $\pi_{1} \widetilde{C}_{\beta}$ is a quotient of $\operatorname{Aut}(\beta)$, so any element in $\pi_{1} \widetilde{C}_{\beta}$ can be represented by a birational automorphism of $X_{\beta}$.

Using Proposition 4.3 we can write down the first two columns of $\widetilde{E}_{*, *}^{1}$, which appear in Figure2,

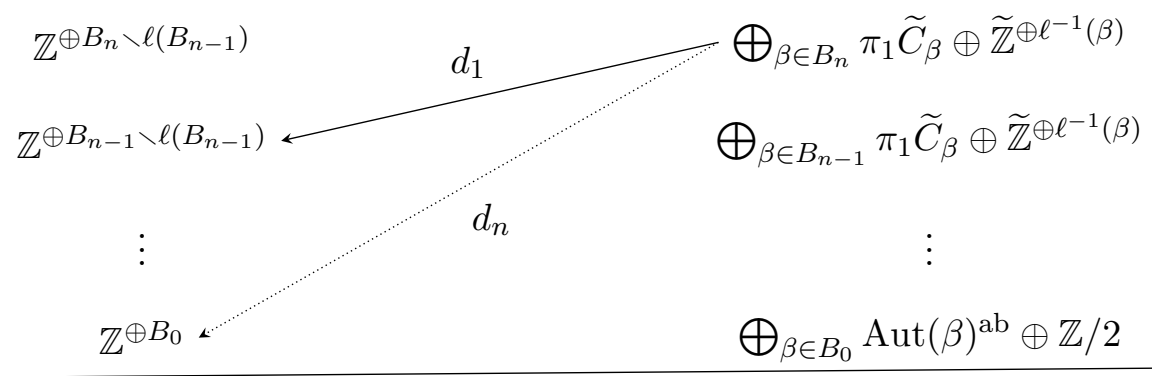

$\pi_{0}$

$\pi_{1}$

Figure 2. Spectral sequence for $K((\mathcal{V} / L)$.)

Lemma 4.6. Let $\beta$ be in $B_{n}$.

(1) For every birational automorphism $\varphi \in \operatorname{Aut}(\beta)$, the homomorphism

$$
\partial: \pi_{1} \widetilde{C}_{\beta} \leq \widetilde{E}_{1, n}^{1} \longrightarrow \widetilde{A}_{0, n-1}
$$

is given by

$$
\partial[\varphi]=\left[X_{\beta} \backslash V\right]-\left[X_{\beta} \backslash U\right]
$$

if $\varphi$ is represented as an isomorphism $U \longrightarrow V$ of open subsets of $X_{\beta}$.

(2) If $\ell^{-1}(\beta)=\emptyset$ then $\partial(\mathbb{Z} / 2)=0$.

(3) Suppose that $\ell^{-1}(\beta) \neq \emptyset$. The homomorphism

$$
\partial: \pi_{1} \operatorname{cofib} \nabla_{\beta} \leq \widetilde{E}_{1, n}^{1} \longrightarrow \widetilde{A}_{0, n-1}
$$

is given by

$$
\partial\left([\alpha]-\left[\alpha^{\prime}\right]\right)=\left[\left(X_{\alpha^{\prime}} \times \mathbb{A}^{1}\right) \backslash V\right]-\left[\left(X_{\alpha} \times \mathbb{A}^{1}\right) \backslash U\right]
$$

for any formal difference $[\alpha]-\left[\alpha^{\prime}\right]$ and for any birational isomorphism $\varphi: X_{\alpha} \times \mathbb{A}^{1} \rightarrow X_{\alpha^{\prime}} \times$ $\mathbb{A}^{1}$ represented by an isomorphism $U \rightarrow V$.

Proof. Proof of (1): The group $\widetilde{E}_{1, n}^{1}$ is $K_{1}\left(\left(\mathcal{V}^{(n)} / L\right) / \widetilde{\iota}\right)$. Since $\left(V^{(n)} / L\right) / \widetilde{\iota} \cong\left(V^{(n)} / \iota\right) / \widetilde{L}$, to compute representatives for its elements it suffices to compute representatives for the elements of $K_{1}\left(\left(\mathcal{V}^{(n)} / \iota\right) / \widetilde{L}\right)$ and check that they represent the correct classes. Since we obtained our calculation of these groups from the long exact sequence for the cofiber sequence

$$
K\left(\mathcal{V}^{(n-1)} / \iota\right) \longrightarrow K\left(\mathcal{V}^{(n)} / \iota\right) \longrightarrow K\left(\left(\mathcal{V}^{(n)} / \iota\right) / \widetilde{L}\right)
$$

it suffices to check that they are correct by checking them in this sequence; thus to represent $[\varphi]$ it suffices to construct a representative of $[\varphi]$ in $K_{1}\left(\mathcal{V}^{(n)} / \iota\right)$ and then compute its image in $K_{1}\left(\left(\mathcal{V}^{(n)} / \iota\right) / \widetilde{L}\right)$. 
By using the model of the cofiber constructed in Theorem 1.12 together with ZakB, Corollary 3.10] we know that the following data represents an element of $E_{1, n}^{1}$. Let $\beta$ be in $B_{n}$ and let $\varphi$ be a birational automorphism of $X_{\beta}$, represented by an isomorphism $U \rightarrow V$ of open subsets of $X_{\beta}$. Then a representative of the class of $\varphi$ in $\pi_{1} \widetilde{C}_{\beta}$ is given by the covering families

$$
\left\{U \longrightarrow X_{\beta}\right\} \cup\left\{X_{\beta} \backslash U \longrightarrow X_{\beta}\right\} \quad \text { and } \quad\left\{U \stackrel{\cong}{\longrightarrow} V \longrightarrow X_{\beta}\right\} \cup\left\{X_{\beta} \backslash V \longrightarrow X_{\beta}\right\} .
$$

Thus $\partial[\varphi]=\left[X_{\beta} \backslash V\right]-\left[X_{\beta} \backslash U\right]$.

Proof of (2): As in part (1), we can construct a representative for the nonzero element of $\mathbb{Z} / 2$ by finding one in $K_{1}\left(\mathcal{V}^{(n)} / \iota\right)$ and then considering its image. Therefore the nonzero element of $\mathbb{Z} / 2$ is represented by two copies of $X_{\beta}$ being swapped, and thus has two complete covering families. Therefore $\partial$ on it is zero.

Proof of (3): To find a representative of $[\alpha]-\left[\alpha^{\prime}\right]$ it suffices to construct an element which maps to $[\alpha]-\left[\alpha^{\prime}\right]$ in $K_{0}\left(\mathcal{V}^{(n-1)} / \iota\right)$ under $\partial$. Thus we can represent it $[\alpha]-\left[\alpha^{\prime}\right]$ by the following data: varieties $X_{\alpha}$ and $X_{\alpha^{\prime}}$, and a birational isomorphism $\varphi: X_{\alpha} \times \mathbb{A}^{1} \rightarrow X_{\alpha^{\prime}} \times \mathbb{A}^{1}$ given as an isomorphism $U \longrightarrow V$. Then by [ZakB, Remark 3.9] the corresponding element of $\pi_{1}$ is represented by the covering families

$$
\left\{U \longrightarrow\left(X_{\alpha} \times \mathbb{A}^{1}\right) \sqcup Z_{\alpha^{\prime}}, Z_{\alpha} \longrightarrow\left(X_{\alpha} \times \mathbb{A}^{1}\right) \sqcup Z_{\alpha^{\prime}}, Z_{\alpha^{\prime}} \longrightarrow\left(X_{\alpha} \times \mathbb{A}^{1}\right) \sqcup Z_{\alpha^{\prime}}\right\}
$$

and

$$
\left\{U \stackrel{\cong}{\longrightarrow} V \longrightarrow\left(X_{\alpha^{\prime}} \times \mathbb{A}^{1}\right) \sqcup Z_{\alpha}, Z_{\alpha} \longrightarrow\left(X_{\alpha^{\prime}} \times \mathbb{A}^{1}\right) \sqcup Z_{\alpha}, Z_{\alpha} \longrightarrow\left(X_{\alpha^{\prime}} \times \mathbb{A}^{1}\right) \sqcup Z_{\alpha}\right\},
$$

where $Z_{\alpha}=\left(X_{\alpha} \times \mathbb{A}^{1}\right) \backslash U$ and $Z_{\alpha^{\prime}}=\left(X_{\alpha^{\prime}} \times \mathbb{A}^{1}\right) \backslash V$ S Then $\partial\left([\alpha]-\left[\alpha^{\prime}\right]\right)=\left[Z_{\alpha^{\prime}}\right]-\left[Z_{\alpha}\right]$.

Proposition 4.7. The map $K(\mathcal{V}) \longrightarrow K(\mathcal{V} / L)$ induces a map of spectral sequences $E_{p, q}^{*} \longrightarrow \widetilde{E}_{p, q}^{*}$. This map is surjective on $\widetilde{E}_{0, n}^{1}$ and surjective onto the component $\bigoplus_{\beta \in B_{n}} \pi_{1} \widetilde{C}_{\beta}$ in $\widetilde{E}_{1, n}^{1}$.

Proof. The first part follows directly from the fact that this map gives rise to a map of filtered spectra. The statement about $\widetilde{E}_{0, n}^{1}$ is true for any such spectral sequence arising from a connective spectrum filtered by connective spectra, since it follows from the fact that for any map of connective spectra $f: X \longrightarrow Y, \pi_{0} Y \longrightarrow \pi_{0}$ cofib $f$ is surjective. The last statement follows by checking that in the map $K_{1}(\mathcal{V}) \longrightarrow K_{1}(\mathcal{V} / L)$ the class of a birational automorphism $\varphi$ maps to itself, as mentioned in the proof of Lemma 4.6 .

\section{Restricting to CONVENIENT FIELDS}

All of the results mentioned so far in the paper are independent of the field $k$ and have thus contained rather little algebraic geometry. We now want to compute some of the differentials in the spectral sequence for $K(\mathcal{V} / L)$. For this we need to restrict our attention to those fields where the computations are feasible.

Definition 5.1. A birational isomorphism $\varphi: X \rightarrow Y$ between smooth projective varieties $X$ and $Y$ of dimension $n$ over $k$ is convenient if

$$
[X \backslash U]-[Y \backslash V] \in \operatorname{im}\left(K_{0}\left(\mathcal{V}^{(n-2)}\right) \stackrel{\cdot \mathbb{L}}{\longrightarrow} K_{0}\left(\mathcal{V}^{(n-1)}\right)\right) .
$$

Here $U \subseteq X$ and $V \subseteq Y$ are open and $\varphi$ is an isomorphism $U \longrightarrow V$.

A field $k$ is convenient if all birational isomorphisms between smooth projective varieties over $k$ are convenient.

\footnotetext{
${ }^{3}$ Rephrasing this in the notation of Z ZakB, Remark 3.9], we use $B_{0}=0, C_{0}=\left\{U, Z_{\alpha}, Z_{\alpha^{\prime}}\right\}, V_{1}=\left(\left\{X_{\alpha} \times\right.\right.$ $\left.\left.\mathbb{A}^{1}, Z_{\alpha^{\prime}}\right\},\left\{X_{\alpha^{\prime}}\right\},\left\{Z_{\alpha}\right\}, 0\right)$ and $W_{1}=\left(\left\{X_{\alpha^{\prime}} \times \mathbb{A}^{1}, Z_{\alpha}\right\},\left\{X_{\alpha}\right\},\left\{Z_{\alpha^{\prime}}\right\}, 0\right)$.
} 
A priori, it is not obvious that the definition of a convenient birational isomorphism is only dependent on the birational isomorphism and not on the choices of $U$ and $V$. In order to check that this is not the case it suffices to check that if $\varphi$ is convenient when represented as an isomorphism $U \rightarrow V$ then it is also convenient when represented as an isomorphism $U^{\prime} \longrightarrow V^{\prime}$ for any open $U^{\prime} \subseteq U$. To see this, observe that

$$
\begin{aligned}
{\left[X \backslash U^{\prime}\right]-\left[Y \backslash V^{\prime}\right] } & =[X \backslash U]+\left[U \backslash U^{\prime}\right]-[Y \backslash V]-\left[V \backslash V^{\prime}\right] \\
& =\underbrace{[X \backslash U]-[Y \backslash V]}_{\in \operatorname{im}(\cdot \mathbb{L})}+\underbrace{\left[U \backslash U^{\prime}\right]-\left[V \backslash V^{\prime}\right]}_{=0} .
\end{aligned}
$$

The equality $\left[U \backslash U^{\prime}\right]=\left[V \backslash V^{\prime}\right]$ is because $\varphi$ restricts to an isomorphism $U \backslash U^{\prime} \longrightarrow V \backslash V^{\prime}$.

All computations in this section are going to be done in convenient fields. Currently, the only fields known to be convenient are fields with characteristic 0. However, as the proof of this (see below) relies only on the Weak Factorization Theorem, any field for which the Weak Factorization Theorem is known is convenient. We introduce this definition to highlight that this is the only property needed for all computations in this section.

Lemma 5.2. Fields with characteristic 0 are convenient.

Proof. If $k$ has characteristic 0 then the Weak Factorization Theorem holds in $k$ Wło09, Section 5.20]. Thus any birational isomorphism $\varphi: X \rightarrow Y$ can be factored as a composition of blowups and blow-downs along smooth centers. We prove that all birational isomorphisms over $k$ are convenient by induction on the number of blowups and blowdowns in this factorization.

We first prove the base case. Suppose that $Y$ is a blowup of $X$ along $Z$. Then in $K_{0}\left(\mathcal{V}_{k}^{(n)}\right)$ we can write $[X]=[U]+[Z]$ and $[Y]=[U]+\left[Z^{\prime}\right]$, where $Z^{\prime}$ is a $\mathbb{P}^{\ell}$ bundle on $Z$ for some $1 \leq \ell \leq n-1-\operatorname{dim} Z$. However, in $K_{0}\left[\mathcal{V}^{(\operatorname{dim} Z+\ell)}\right]$ and thus in $K_{0}\left[\mathcal{V}^{(n-1)}\right],\left[Z^{\prime}\right]=\left[Z \times \mathbb{P}^{\ell}\right]$. Thus

$$
\begin{aligned}
{[X \backslash U]-[Y \backslash U] } & =[Z]-\left[Z \times \mathbb{P}^{\ell}\right]=[Z]-\left([Z]+[Z] \mathbb{L}+\cdots+[Z] \mathbb{L}^{\ell}\right) \\
& =-\mathbb{L}\left([Z]+\cdots+[Z] \mathbb{L}^{\ell-1}\right) \in \mathbb{L} K_{0}\left(\mathcal{V}^{(n-2)}\right) .
\end{aligned}
$$

For a blowdown the computation is the same with a reversed sign.

Now we prove the induction step. Suppose that $\varphi=\varphi^{\prime \prime} \varphi^{\prime}$, where $\varphi^{\prime}$ and $\varphi^{\prime \prime}$ require fewer blowups/blowdowns than $\varphi$. Represent $\varphi^{\prime}: X \rightarrow Y$ by $U \stackrel{\cong}{\longrightarrow} U^{\prime}$ and $\varphi^{\prime \prime}: Y \rightarrow Z$ by $V \stackrel{\cong}{\longrightarrow} V^{\prime}$. By the induction hypothesis both $\varphi^{\prime}$ and $\varphi^{\prime \prime}$ are convenient.

Suppose first that $U^{\prime}=V$. Then $\varphi$ can be represented as an isomorphism $U \longrightarrow V^{\prime}$, and we have

$$
\left[Z \backslash V^{\prime}\right]-[X \backslash U]=\left(\left[Z \backslash V^{\prime}\right]-[Y \backslash V]\right)+\left(\left[Y \backslash U^{\prime}\right]-[X \backslash U]\right) \in \operatorname{im}(\cdot \mathbb{L}) .
$$

Thus in this case $\varphi$ is convenient.

Now consider the general case. Let $W=\varphi^{\prime}(U) \cap V \subseteq Y$. Then $\varphi^{\prime}$ can be represented as an isomorphism $\left(\varphi^{\prime}\right)^{-1}(W) \longrightarrow W$ and $\varphi^{\prime \prime}$ can be represented as an isomorphism $W \longrightarrow \varphi^{\prime \prime}(W)$. This reduces to the above case, and we see that $\varphi$ must also be convenient.

Definition 5.3. We define the line degree of a birational isomorphism class $\alpha$ in $B_{n}$, denoted $\operatorname{deg} \alpha$ to be the maximum integer $s$ such that $\alpha$ in $\ell^{s}\left(B_{n-s}\right)$. We define the equivalence relation $\sim_{r}$ on $B_{n}$ by defining $\alpha \sim_{r} \alpha^{\prime}$ if $\ell^{r}(\alpha)=\ell^{r}\left(\alpha^{\prime}\right)$; we write $\alpha \sim_{\infty} \alpha^{\prime}$ if there exists $r$ such that $\alpha \sim_{r} \alpha^{\prime}$.

By definition, $\alpha$ and $\alpha^{\prime}$ in $B_{n}$ are stably birational if and only if $\alpha \sim_{\infty} \alpha^{\prime}$.

Our first result computes the differentials in the spectral sequence for $K(\mathcal{V} / L)$ in a more convenient form than that given by Lemma 4.6 .

Lemma 5.4. Let $k$ be a convenient field, and let $\beta$ be in $B_{n}$. Consider the spectral sequence $\widetilde{E}_{p, q}^{*}$.

(1) For every birational automorphism $\varphi$ in $\operatorname{Aut}(\beta)$ and all $r \geq 1, d_{r}[\varphi]=0$.

(2) If $\ell^{-1}(\beta)=\emptyset$ then $d_{r}(\mathbb{Z} / 2)=0$ for all $r \geq 1$. 
(3) Suppose $\ell^{-1}(\beta) \neq \emptyset$. For distinct $\alpha, \alpha^{\prime}$ in $\ell^{-1}(\beta)$,

$$
d_{r}\left([\alpha]-\left[\alpha^{\prime}\right]\right)= \begin{cases}0 & 1 \leq r \leq \min \left(\operatorname{deg} \alpha, \operatorname{deg} \alpha^{\prime}\right) \\ {\left[\left(\ell^{r-1}\right)^{-1}(\alpha)\right]-\left[\left(\ell^{r-1}\right)^{-1}\left(\alpha^{\prime}\right)\right]} & r=\min \left(\operatorname{deg} \alpha, \operatorname{deg} \alpha^{\prime}\right)+1 .\end{cases}
$$

Here, $\left(\ell^{r-1}\right)^{-1}(\alpha)$ is any preimage of $\alpha$ under $\ell^{r-1}$.

(4) For all $r \geq 1$,

$$
\widetilde{E}_{0, n}^{r} \cong \mathbb{Z}^{\oplus\left(B_{n} / \sim_{r-1}\right) \backslash \ell\left(B_{n-1}\right)} .
$$

(5) For $\alpha, \alpha^{\prime}$ as in part (3), $d_{\min \left(\operatorname{deg} \alpha, \operatorname{deg} \alpha^{\prime}\right)+1}\left([\alpha]-\left[\alpha^{\prime}\right]\right) \neq 0$.

In particular, the formula in (3) and the statement in (5) tell us that the length of a differential killing a difference $[\alpha]-\left[\alpha^{\prime}\right]$ gives us the "minimal stability degree" of $\alpha$ and $\alpha^{\prime}$ : if $d_{r}$ hits $[\alpha]-\left[\alpha^{\prime}\right]$ then the birational isomorphism classes $\alpha$ and $\alpha^{\prime}$ are birational after multiplication with $\mathbb{P}^{r}$ but not after multiplication with $\mathbb{P}^{r-1}$.

Proof. Proof of (1): The differentials in the spectral sequence are obtained from the boundary map in the long exact sequence. Thus to show that $d_{r}[\varphi]=0$ for all $r$ it suffices to show that $\partial[\varphi]$ is 0 , where $\widetilde{\iota}: \mathcal{V}^{(n-1)} / L \longrightarrow \mathcal{V}^{(n)} / L$ and $\partial$ is the connecting homomorphism

$$
K_{1}\left(\left(\mathcal{V}^{(n)} / L\right) / \widetilde{\iota} \stackrel{\partial}{\longrightarrow} K_{0}\left(\mathcal{V}^{(n-1)} / L\right)\right.
$$

in the long exact sequence in homotopy of the cofiber sequence

$$
K\left(\mathcal{V}^{(n-1)} / L\right) \longrightarrow K\left(\mathcal{V}^{(n)} / L\right) \longrightarrow K\left(\left(\mathcal{V}^{(n)} / L\right) \widetilde{\iota}\right)
$$

From Lemma 4.6 and the fact that $k$ is convenient we know that

$$
\partial[\varphi]=\left[X_{\beta} \backslash V\right]-\left[X_{\beta} \backslash U\right]
$$

is in $\mathbb{L} K_{0}\left(\mathcal{V}^{(n-2)}\right)$. However,

$$
K_{0}\left(\mathcal{V}^{(n-1)} / L\right) \cong K_{0}\left(\mathcal{V}^{(n-1)}\right) / \mathbb{L} K_{0}\left(\mathcal{V}^{(n-2)}\right),
$$

so we see that $\partial[\varphi]=0$.

Proof of (2): By Lemma 2.1 it suffices to show that $\partial(\mathbb{Z} / 2)=0$. This is proved in Lemma 4.6(2). Proof of (3): From lemma 4.6, we know that

$$
\partial\left([\alpha]-\left[\alpha^{\prime}\right]\right)=\left[\left(X_{\alpha^{\prime}} \times \mathbb{A}^{1}\right) \backslash V\right]-\left[\left(X_{\alpha} \times \mathbb{A}^{1}\right) \backslash U\right]
$$

for any birational isomorphism $\varphi: X_{\alpha} \times \mathbb{A}^{1} \rightarrow X_{\alpha^{\prime}} \times \mathbb{A}^{1}$. Note that we can think of $\varphi$ as a birational isomorphism $X_{\alpha} \times \mathbb{P}^{1} \rightarrow X_{\alpha^{\prime}} \times \mathbb{P}^{1}$; then since $k$ is convenient we know that

$$
\left[\left(X_{\alpha^{\prime}} \times \mathbb{P}^{1}\right) \backslash V\right]-\left[\left(X_{\alpha} \times \mathbb{P}^{1}\right) \backslash U\right]
$$

is in $\mathbb{L} K_{0}\left(\mathcal{V}^{(n-2)}\right)$. Since $\left(X_{\alpha^{\prime}} \times \mathbb{P}^{1}\right) \backslash V=\left(\left(X_{\alpha^{\prime}} \times \mathbb{A}^{1}\right) \backslash V\right) \uplus X_{\alpha^{\prime}}$ we conclude that in $K_{0}\left(\mathcal{V}^{(n-1)}\right)$

$$
\left[\left(X_{\alpha^{\prime}} \times \mathbb{A}^{1}\right) \backslash V\right]-\left[\left(X_{\alpha} \times \mathbb{A}^{1}\right) \backslash U\right] \equiv\left[X_{\alpha}\right]-\left[X_{\alpha^{\prime}}\right]\left(\bmod \mathbb{L} K_{0}\left(\mathcal{V}^{(n-2)}\right)\right) .
$$

Thus we have shown that $\partial\left([\alpha]-\left[\alpha^{\prime}\right]\right)=\left[X_{\alpha}\right]-\left[X_{\alpha^{\prime}}\right]$. Now suppose that there exists an $s$ such that $\alpha=\ell^{s}(\gamma)$ and $\alpha^{\prime}=\ell^{s}\left(\gamma^{\prime}\right)$. Since $X_{\alpha}, X_{\alpha^{\prime}}$ are arbitrary smooth projective representatives of the birational isomorphism classes we could have chosen $X_{\alpha}=X_{\gamma} \times \mathbb{P}^{s}$ and $X_{\alpha^{\prime}}=X_{\gamma^{\prime}} \times \mathbb{P}^{s}$. Then $\left[X_{\alpha}\right]-\left[X_{\alpha^{\prime}}\right] \equiv\left[X_{\gamma}\right]-\left[X_{\gamma^{\prime}}\right]\left(\bmod \mathbb{L} K_{0}\left(\mathcal{V}^{(n-2)}\right)\right)$.

Proof of $(4,5)$ : In order to prove (5) it suffices to prove the following statement for all $r$ :

(5a) If $\alpha \neq \alpha^{\prime}$ in $\ell^{-1}(\beta)$ and $\min \left(\operatorname{deg} \alpha, \operatorname{deg} \alpha^{\prime}\right)<r$ then $d_{\min \left(\operatorname{deg} \alpha, \operatorname{deg} \alpha^{\prime}\right)+1}\left([\alpha]-\left[\alpha^{\prime}\right]\right) \neq 0$. 
We prove (4) and (5a) by a paired induction on $r$. For $r=1$, statement (4) is just the computation of $\widetilde{E}_{0, n}^{1}$. Statement (5a) just says that if one of $\alpha, \alpha^{\prime}$ is not in $\ell\left(B_{n-1}\right)$ then $[\alpha]-\left[\alpha^{\prime}\right] \neq 0$, which follows from the formula for $\widetilde{E}_{0, n}^{1}$. Now suppose that both statements are true up to $r$ and consider $d_{r}$. By the induction hypothesis we know that for all $m$

$$
\widetilde{E}_{0, m}^{r} \cong \mathbb{Z}^{\oplus\left(B_{m} / \sim_{r-1}\right) \backslash \ell\left(B_{m-1}\right)} .
$$

Note that the free part of $\widetilde{E}_{1, n}^{r}$ is generated by all $\alpha$ in $\ell^{-1}(\beta)$ such that $\operatorname{deg} \alpha \geq r-1$, since by (5a) all differences where at least one term had degree less than $r-1$ had a nonzero differential on it. Thus to prove (4) we need to show that the cokernel of

$$
d_{r}: \bigoplus_{\substack{\beta \in B_{n} \\ \ell^{-1}(\beta) \neq \emptyset}} \widetilde{\mathbb{Z}}^{\oplus\left\{\alpha \in \ell^{-1}(\beta) \mid \operatorname{deg} \alpha \geq r-1\right\}} \longrightarrow \mathbb{Z}^{\oplus\left(B_{n-r} / \sim_{r-1}\right) \backslash \ell\left(B_{n-r-1}\right)}
$$

is $\mathbb{Z}^{\oplus\left(B_{n-r} / \sim_{r}\right) \backslash \ell\left(B_{n-r-1}\right)}$. (Note that restricting to those $\beta$ with nonzero preimage doesn't change the cokernel; we do this purely for convenience.) For any $\beta$, suppose that $\alpha, \alpha^{\prime} \in \ell^{-1}(\beta)$ are distinct. If $\operatorname{deg} \alpha, \operatorname{deg} \alpha^{\prime}>r-1$ then by $(3) d_{r}\left([\alpha]-\left[\alpha^{\prime}\right]\right)=0$, so they do not influence the cokernel of $d_{r}$. Thus it suffices to consider those pairs $\alpha, \alpha^{\prime}$ with $\operatorname{deg} \alpha=r-1$.

Let $\gamma$ be in $\left(\ell^{r-1}\right)^{-1}(\alpha)$ and $\gamma^{\prime}$ be in $\left(\ell^{r-1}\right)^{-1}\left(\alpha^{\prime}\right)$. Note that by definition $\gamma \sim_{r} \gamma^{\prime}$ but $\gamma \chi_{r-1} \gamma^{\prime}$. On the other hand, if $\gamma \sim_{r} \gamma^{\prime}$ but $\gamma \chi_{r-1} \gamma^{\prime}$ then

$$
d_{r}\left(\left[\ell^{r-1}(\gamma)\right]-\left[\ell^{r-1}\left(\gamma^{\prime}\right)\right]\right)=[\gamma]-\left[\gamma^{\prime}\right],
$$

so any such pair is in the image of $d_{r}$. Thus the image of $d_{r}$ quotients out by $[\gamma]-\left[\gamma^{\prime}\right]$ for those pairs $\gamma$ such that $\gamma \chi_{r-1} \gamma^{\prime}$ but $\gamma \sim_{r} \gamma^{\prime}$, and we see that

$$
\widetilde{E}_{0, n-r}^{r+1}=\mathbb{Z}^{\oplus\left(B_{n-r} / \sim_{r}\right) \backslash \ell\left(B_{n-r-1}\right)} .
$$

It remains to check (5a): if $\operatorname{deg} \alpha=r-1$ and $\operatorname{deg} \alpha^{\prime} \geq r-1$ then $d_{r}\left([\alpha]-\left[\alpha^{\prime}\right]\right) \neq 0$. Suppose that the opposite held, so that $[\gamma]-\left[\gamma^{\prime}\right]=0$. Then from (4) we know that $\gamma \sim_{r-1} \gamma^{\prime}$, so that $\alpha=\ell^{r-1}(\gamma)=\ell^{r-1}\left(\gamma^{\prime}\right)=\alpha^{\prime}$, a contradiction. Statement (5a) for $r$ follows.

Lemma 5.4 directly implies the following:

Corollary 5.5. The homomorphism $E_{1, n}^{1} \longrightarrow \widetilde{E}_{1, n}^{1}$ is surjective on permanent cycles.

As a quick application of Lemma 5.4 we prove an extension of Liu and Sebag's result [LS10, Corollary 5(1)].

Corollary 5.6. A variety $X$ has stable dimensional complexity $k$ if $k$ is the minimal integer such that there exists a variety $X^{\prime}$ of dimension $k$ such that $X$ is stably birational to $X^{\prime} \times \mathbb{A}^{\operatorname{dim} X-k}$. If $X$ and $Y$ both have dimension $n$ and stable dimensional complexity $n$ and $[X]=[Y]$ then they are stably birational.

Proof. Let $n=\operatorname{dim} X$. If $X$ and $Y$ are birational then we are done. If they are not birational then in the spectral sequence for $K(\mathcal{V})$ we have $[X] \neq[Y]$ in $E_{0, n}^{1}$, but $[X]=[Y]$ in $E_{0, n}^{\infty}$. Let $r$ be the minimal integer such that $[X]=[Y]$ in $E_{0, n}^{r}$. Projecting down to $\widetilde{E}_{0, n}^{r}$ we see that $[X]=[Y]$ there as well. If $[X] \neq 0$ then by Lemma 5.4 (4) we must have $X \sim_{r-1} Y$, and $X$ and $Y$ are stably birational, as desired. On the other hand, if $[X]=0$ then by Lemma 5.4(4) there exists a birational isomorphism class $\alpha \in \ell\left(B_{n-1}\right)$ such that $X \times \mathbb{A}^{r-1} \in \ell^{r-1}(\alpha)$; in this case $X$ has stable dimensional complexity less than $n$, contradicting the assumption in the corollary. Thus this cannot happen and $X$ and $Y$ are stably birational, as desired.

Note that this does not contradict Borisov's construction in [Bor]: in that paper, Borisov constructs two varieties $X$ and $Y$ which are not stably birational but which have $[X]=[Y]$. However, the fact that $[X]=[Y]$ is shown by having $[X]=\left[X^{\prime}\right] \mathbb{L}^{7}$ and $[Y]=\left[Y^{\prime}\right] \mathbb{L}^{7}$, with no consideration 
of whether 7 is the smallest power of $\mathbb{L}$ necessary for the result. Borisov shows that the power of $\mathbb{L}$ must be at least 1 (and therefore that multiplication by $\mathbb{L}$ has a kernel); this corollary is another proof of that fact, since if $\left[X^{\prime}\right]=\left[Y^{\prime}\right]$ then $X^{\prime}$ and $Y^{\prime}$ (and therefore $X$ and $Y$ ) would have to be stably birational.

We are now ready to prove Theorem E over a convenient field.

Proof of Theorem E. By Lemma 5.4(4) we know that $\widetilde{E}_{0, n}^{\infty} \cong \mathbb{Z}^{\oplus\left(B_{n} / \sim_{\infty}\right) \backslash \ell\left(B_{n-1}\right)}$. Since the groups in the 0 column are free, this means that

$$
K_{0}(\mathcal{V}) /(\mathbb{L}) \cong K_{0}(\mathcal{V} / L) \cong \bigoplus_{n \geq 0} \widetilde{E}_{0, n}^{\infty} \cong \bigoplus_{n \geq 0} \mathbb{Z}^{\oplus\left(B_{n} / \sim_{\infty}\right) \backslash \ell\left(B_{n-1}\right)}
$$

We claim that this contains one copy of $\mathbb{Z}$ for each stable birational isomorphism class.

From the analysis in Lemma 5.4 this contains at least one copy of $\mathbb{Z}$ for each stable birational isomorphism class, since there is at least one at $\widetilde{E}^{1}$ and the differentials only quotient out differences between stably birational varieties. Thus we just need to check that at $E^{\infty}$ only one representative of each class is left. From the formula in part (4) we have quotiented out by stable birational equivalence in each degree, so we just need to check that we also quotient out the difference in different degrees.

Let $\eta$ be a stable birational isomorphism class, and let $X$ be a representative of this stable birational isomorphism class of minimal dimension; write $m=\operatorname{dim} X$. We claim that if $\alpha$ in $B_{n}$ satisfies $\ell^{n-m+r}([X])=\ell^{r}(\alpha)$ then $[\alpha]=0$ in $\widetilde{E}_{0, n}^{\infty}$. For convenience, let us suppose that $r$ is minimal. Then inside $\widetilde{E}_{1, n-m+r}^{1}$ we have a nonzero element represented by $\left[\ell^{n-m+r-1}([X])\right]-\left[\ell^{r-1}(\alpha)\right]$, and

$$
d_{r-1}\left(\left[\ell^{(n-m+r-1)}([X])\right]-\left[\ell^{r-1}(\alpha)\right]\right)=\left[\ell^{n-m}([X])\right]-[\alpha]=-[\alpha] \in \widetilde{E}_{0, n}^{r-1},
$$

since $m<n$. Thus $[\alpha]=0$ in $\widetilde{E}_{0, n}^{r}$ and therefore also in $\widetilde{E}_{0, n}^{\infty}$. The only representative of the stable birational isomorphism class $\eta$ that survives to $\widetilde{E}^{\infty}$ is $[X]$.

In addition, from this proof we see that every generator in $E_{0, n}^{\infty}$ is a representative of a stable birational isomorphism class whose minimal representative appears in dimension $n$. Thus the spectral sequence for $K(\mathcal{V} / L)$ keeps track not only of "minimal stability degree", but also of the "minimal dimension" of a stable birational isomorphism class.

Proof of Theorem $\mathrm{C}$. We prove the contrapositive of this theorem: if all $\psi_{n}$ have trivial kernels then $\mathbb{L}$ is not a zero divisor.

The cofiber sequence $K(\mathcal{V}) \stackrel{K(L)}{\longrightarrow} K(\mathcal{V}) \longrightarrow K(\mathcal{V} / L)$ gives rise to a long exact sequence in homotopy

$$
K_{1}(\mathcal{V}) \longrightarrow K_{1}(\mathcal{V} / L) \longrightarrow K_{0}(\mathcal{V}) \stackrel{\cdot \mathbb{L}}{\longrightarrow} K_{0}(\mathcal{V}) .
$$

Thus the kernel of multiplication by $\mathbb{L}$ is equal to the cokernel of the map $K_{1}(\mathcal{V}) \longrightarrow K_{1}(\mathcal{V} / L)$. We therefore want to analyze the cokernel of the map $E_{1, q}^{\infty} \longrightarrow \widetilde{E}_{1, q}^{\infty}$. From the computation of the differentials in Lemma 5.4 we see that

$$
\widetilde{E}_{1, n}^{\infty} \cong\left[\left(\bigoplus_{\beta \in B_{n}} \pi_{1} \widetilde{C}_{\beta}\right) \oplus \mathbb{Z} / 2^{\oplus B_{n} \backslash \ell\left(B_{n-1}\right)}\right],
$$

where the square brackets denote some quotient of the given group. By Corollary $5.5 E_{1, n}^{1} \longrightarrow \widetilde{E}_{1, n}^{1}$ is surjective on this component.

Suppose that all birational automorphisms of varieties extend to piecewise automorphisms. Then by Theorem 3.4 all differentials between the 1 -st and 0 -th columns of the spectral sequence are 0 . Since the map between spectral sequences is surjective on all pages on the component of the $E^{1}$ page that survives to $E^{\infty}$, and all differentials out of this component are zero, the map on $E^{\infty}$ 
pages is also surjective, and therefore the map $K_{1}(\mathcal{V}) \longrightarrow K_{1}(\mathcal{V} / L)$ is surjective; thus $\cdot \mathbb{L}$ is injective and $\mathbb{L}$ is not a zero divisor.

By doing a little bit more spectral sequence analysis, we can finally prove Theorem D.

Proof of Theorem $D$. Pick a representative $x$ of minimal filtration degree $n$ in the preimage of $\chi$ in $K_{1}(\mathcal{V} / L)$. Then in the long exact sequence

$$
K_{1}\left(\mathcal{V}^{(n)}\right) \longrightarrow K_{1}\left(\mathcal{V}^{(n)} / L\right) \stackrel{\partial}{\longrightarrow} K_{0}\left(\mathcal{V}^{(n-1)}\right) \stackrel{\cdot \mathbb{L}}{\longrightarrow} K_{0}\left(\mathcal{V}^{(n)}\right)
$$

write $\partial[x]=[X]-[Y]$, with $X$ and $Y$ of minimal dimension. We know that $\operatorname{dim} X=\operatorname{dim} Y$ because $\left[X \times \mathbb{A}^{1}\right]=\left[Y \times \mathbb{A}^{1}\right]$ and this means that $\operatorname{dim} X+1=\operatorname{dim} Y+1$ by [LS10, Corollary 5]. We claim that $\operatorname{dim} X<n-1$. Indeed, consider the commutative diagram:

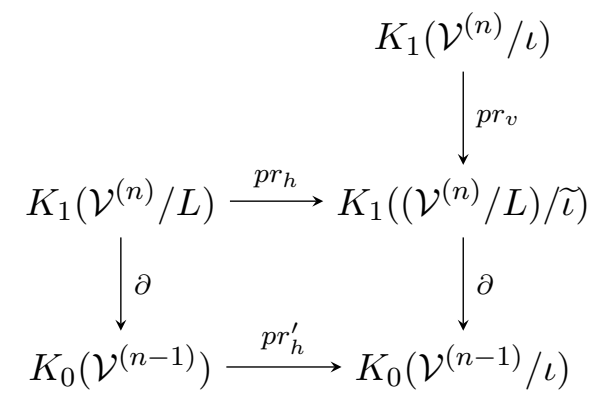

where the two columns are obtained from the long exact sequences in homotopy of the two righthand columns of Diagram 4.5 and the horizontal morphisms are induced by the morphisms in the rows of the diagram. From our choice of $n, x$ is in $K_{1}\left(\mathcal{V}^{(n)} / L\right)$ and $p r_{h}(x) \neq 0$; since $x$ is a permanent cycle in the spectral sequence for $K(\mathcal{V} / L)$, by Corollary 5.5 $p r_{h}(x)$ is in the image of $p r_{v}$; thus $\partial\left(p r_{h}(x)\right)=0$. On the other hand, $\partial\left(p r_{h}(x)\right)=p r_{h}^{\prime}(\partial(x))=\left(p r_{h}^{\prime}\right)([X]-[Y])$.

Since $K_{0}\left(\mathcal{V}^{(n-1)} / \iota\right)$ is the free abelian group on $B_{n-1}$ and $p r_{h}^{\prime}([X]-[Y])=0$ this means that the components of $X$ and $Y$ of dimension $n-1$ are pairwise birational. In particular, since $X$ and $Y$ were chosen to have minimal dimension, this means that $\operatorname{dim} X<n-1$.

Now consider the commutative diagram

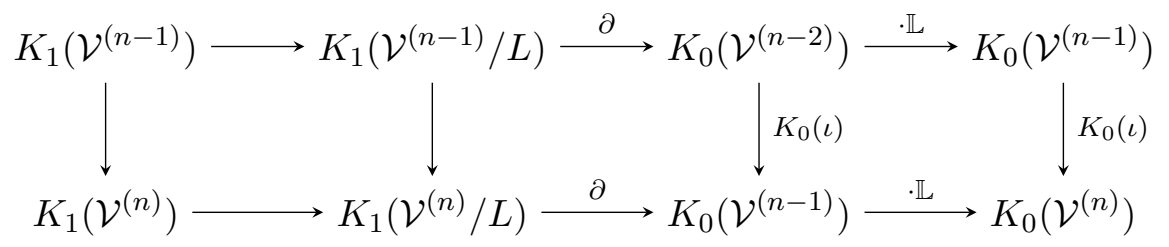

where the top and bottom rows are exact. Then $[X]-[Y]$ lives in $K_{0}\left(\mathcal{V}^{(n-2)}\right)$, but it is not in the image of $\partial$, since the first filtration degree that contains $x$ is $n$. Thus $\mathbb{L}([X]-[Y]) \neq 0$. However, $K_{0}(\iota)([X]-[Y])$ is in the image of $\partial$, so $\mathbb{L}\left(K_{0}(\iota)([X]-[Y])\right)=0$; thus $\mathbb{L}([X]-[Y])$ is in the kernel of $K_{0}(\iota)$, and thus of $\psi_{n}$. Therefore $\left[X \times \mathbb{A}^{1}\right]=\left[Y \times \mathbb{A}^{1}\right]$ in $K_{0}(\mathcal{V})$ but they are not piecewise isomorphic.

We conclude this paper with the following conjecture, which attempts to correct Question 1.

Conjecture. Suppose that $X$ and $Y$ are varieties over a convenient field $k$ such that $[X]=[Y]$ in $K_{0}\left(\mathcal{V}_{k}\right)$. Then there exist varieties $X^{\prime}$ and $Y^{\prime}$ such that $\left[X^{\prime}\right] \neq\left[Y^{\prime}\right],\left[X^{\prime} \times \mathbb{A}^{1}\right]=\left[Y^{\prime} \times \mathbb{A}^{1}\right]$, and $X \amalg\left(X^{\prime} \times \mathbb{A}^{1}\right)$ is piecewise isomorphic to $Y \amalg\left(Y^{\prime} \times \mathbb{A}^{1}\right)$. 
This conjecture says that elements in the kernel of multiplication by $\mathbb{L}$ are the only possible "errors" to equality in the Grothendieck ring implying that varieties are piecewise isomorphic. Note that the definition of a convenient field is the statement that this is the case for inclusions $K_{0}\left(\mathcal{V}^{(n)}\right) \longrightarrow K_{0}\left(\mathcal{V}^{(n+1)}\right)$. In addition, Corollary 5.6 states that if $[X]=[Y]$ for certain $X$ and $Y$ then $X$ and $Y$ are stably birational: that the error to their being birational to begin with is a power of $\mathbb{L}$.

\section{REFERENCES}

[Boa99] J. Michael Boardman. Conditionally convergent spectral sequences. In Homotopy invariant algebraic structures (Baltimore, MD, 1998), volume 239 of Contemp. Math., pages 49-84. Amer. Math. Soc., Providence, RI, 1999.

[Bor] Lev Borisov. Class of the affine line is a zero divisor in the Grothendieck ring. http://arxiv.org/pdf/1412.6194.

[DL99] Jan Denef and François Loeser. Germs of arcs on singular algebraic varieties and motivic integration. Invent. Math., 135(1):201-232, 1999.

[Gro99] M. Gromov. Endomorphisms of symbolic algebraic varieties. J. Eur. Math. Soc. (JEMS), 1(2):109-197, 1999.

[Hov99] Mark Hovey. Model categories, volume 63 of Mathematical Surveys and Monographs. American Mathematical Society, Providence, RI, 1999.

[Joh02] Peter T. Johnstone. Sketches of an elephant: a topos theory compendium. Vol. 2, volume 44 of Oxford Logic Guides. The Clarendon Press Oxford University Press, Oxford, 2002.

[Kar] Ilya Karzhemanov. On the cut-and-paste property of algebraic varieties. http://arxiv.org/abs/1411.6084.

[LL03] Michael Larsen and Valery A. Lunts. Motivic measures and stable birational geometry. Mosc. Math. J., 3(1):85-95, 259, 2003.

[LS10] Qing Liu and Julien Sebag. The Grothendieck ring of varieties and piecewise isomorphisms. Math. Z., 265(2):321-342, 2010.

[LS12] Stéphane Lamy and Julien Sebag. Birational self-maps and piecewise algebraic geometry. J. Math. Sci. Univ. Tokyo, 19(3):325-357, 2012.

[McC01] John McCleary. A user's guide to spectral sequences, volume 58 of Cambridge Studies in Advanced Mathematics. Cambridge University Press, Cambridge, second edition, 2001.

[MMSS01] M. A. Mandell, J. P. May, S. Schwede, and B. Shipley. Model categories of diagram spectra. Proc. London Math. Soc. (3), 82(2):441-512, 2001.

[Poo02] Bjorn Poonen. The Grothendieck ring of varieties is not a domain. Math. Res. Lett., 9(4):493-497, 2002.

[Rie14] Emily Riehl. Categorical homotopy theory, volume 24 of New Mathematical Monographs. Cambridge University Press, Cambridge, 2014.

[Wło09] Jarosław Włodarczyk. Simple constructive weak factorization. In Algebraic geometry-Seattle 2005. Part 2, volume 80 of Proc. Sympos. Pure Math., pages 957-1004. Amer. Math. Soc., Providence, RI, 2009.

[ZakA] Inna Zakharevich. The $K$-theory of assemblers. http://arxiv.org/abs/1401.3712.

[ZakB] Inna Zakharevich. On $K_{1}$ of an assembler. http://arxiv.org/abs/1506.06197. 